\title{
Efficacité de Weblog sur le développement des concepts de la recherche en sciences de l'éducation chez les étudiants des études supérieures à la faculté de pédagogie.
}

\section{Dr. Maha Abdel-Ghany Abdel-Fattah El-seify}

\section{Sommaire.}

L'Internet a tenu sa promesse de révolution numérique.Accessible dans les lieux publics (bibliothèques, écoles, espaces publics numérique,cybercafé) et a également fait son entrée dans de nombreux foyers devenant par ricochet un nouvel espace social, un lieu de communication et d'échange, un point de rencontre.Les weblogs participent à cette aspiration d'interconnexion.Ils représentent un espace de libre expression et rendre possible la prise de parole publique des internautes. Les blogs occupent aujourd'hui une place centrale dans notre accès quotidien à l'information.A contrario des sites web,les webblogs sont accessibles au plus grand nombre,simples à mettre en ouvre, plus rapides à installer.Le webblog,terme anglophone que l'on trouve usuellement sous forme contractée blog signifie " trace sur le Web". Un Weblog est un blocnotes, un carnet de bord personnel,un joural en ligne.On l'appelle aussi carnet web ou joueb (contraction de "journal web").Le blog permet de partager des informations et des fichiers en les mettant à disposition des internautes. Cet outil de publication autorise une dynamicité beaucoup plus importante qu'un site web traditionnel.IL est interactif;l'internaute lecteur peut commenter les écrits du blog via un formulaire en ligne et ce jeu de vaet-vient permet d'établir une communication entre l'auteur et ses lecteurs, créant ainsi une véritable communauté virtuelle. Le blogue a permis d'assurer la dynamique du groupe en développant un esprit de promotion et en favorisant le partage d'expériences et de savoir-faire; le blogue a permis une réflexion plus construite et individuelle. D'autre part, le travail de recherche est un travail collectif:toutes les occasions doivent être saisies pour s'associer à d'autres et faire partie de groupes de travail.Le chercheur doit être capable d'expliquer la manière dont il a produit ces connaissances.IL va devoir répondre à de nombreuses questions lui demandant de justifier ce qu'il avance.IL faut donc qu'il adopte progressivement une certaine attitude à l'égard de ce qu'il avance et des moyens qu'il a utilisés pour y parvenir.Il mène en permanence un travail de réflexion sur sa propre démarche (on appelle cela la "réflexivité") et peut à tout moment dire où il en est et quelle méthode il utilise.Le chercheur indique les fondements, les points de départ, les postulats sur lesquels il fait reposer son travail. Il définit précisément les notions qu'il utilise.Du rest,il questionne et justifie les choix (de méthodes ou de contenu) qu'il a dî faire(Ce que certains appellent le "travail épistémique").( Livian ,2015,14) Pour parvenir à ce niveau de la "réflexivité", le chercheur doit maîtriser les fondements, les concepts et compétences de la recherche en sciences de l'éducation. D'où vient l'idée de cette étude. L'objectif de cette étude est

\section{3}

Journal of Arabic Studies in Education \& Psychology(ASEP) 
d'utiliser le weblog en vue de développer quelques concepts de la recherche en sciences de l'éducation. L'échantillon représentatif de l'étude était 30 chercheurs du diplôme professionnel qui étudient la matière «Fondements de recherche ", à la faculté de pédagogie de Menoufya (l'année universitaire 2015/2016).Les données ont été recueillies à partir des tâches concernant la recherche. C'est les méthodes de recherche descriptive et expérimentale qui ont été employées dans cette étude. Les outils de cette étude sont : Une liste des concepts-clés de recherche en éducation; Un test des concepts de recherche en éducation en vue d'évaluer les niveaux des chercheurs des facultés de pédagogie; On utilise le blog concernant les concepts fondamentaux en sciences de l'éducation en vue d'améliorer les niveaux des chercheurs des facultés de pédagogie. Les résultats de l'étude montrent que le blogue proposé a une grande efficacité sur le développement des concepts de recherche en sciences de l'éducation.En effet, le succès de cette expérience a tenu au fait que presque tous les étudiants du diplôme professionnel, se voient prêter un ordinateur portable, ainsi qu'une clé USB et un sac à dos. Le blogue a permis une réflexion plus construite et individuelle des travaux menés pendant le cour, et constitué une aide à la rédaction du mémoire de recherche. Les résultats sont en accord avec ceux d'autres auteurs comme Lebrun $(2005,186)$ qui affirme que "les bénéfices les plus flagrants de l'intégration des technologies de l'information et de la communication dans l'enseignement sont à rechercher parmi les méthodes ouvertes et actives [dont font partie le blogue], car elles contribuent à la préparation des personnalités "fortes" que la société revendique».

\section{Première Partie: Préliminaire du problème}

\section{Position du problème:}

Radi explique le déclin de motivation chez les étudiants et les chercheurs en Egypte. Actuellement, Le fonctionnement de l'université est très critiqué en Egypte, tant en ce qui concerne l'infrastructure que l'enseignement. Les enseignants représentent la principale et parfois la seule source d'apprentissage pour les étudiants, le problème de matériel est bien sûr centrale dans la formation à la recherche, et dans la pratique de cette dernière (Radi ,2001,23)

D'autre part ,Plusieurs auteurs, comme Batier (2005), Journot,Lelièvre\& Diolosa (2006), Lebrun (2002\&2005),(Tomé,2007) affirment l'intérêt et les nombreux avantages ,dans le contexte universitaire, du blogue, présenté comme cahier de laboratoire virtuel, inséré dans une plateforme e-learning, le blogue permet d'offrir un 
apprentissage à distance. Cet outil est une brique pédagogique. Il en permet de mettre en forme le texte des billets et gère les commentaires associés.

Afin d'être sûr que l'étude actuelle est nécessaire dans le cadre du processus de l'enseignement à l'université , la chercheuse a préparé un test pour constater le degré de maîtrise de quelques concepts de base de recherches en sciences de l'éducation chez un groupe des étudiants de diplôme professionnel de la faculté de pédagogie de Menoufya. On a déterminé, dans ce teste, des concepts de base de recherches en sciences de l'éducation d'après les écrits et les études antérieures. Ces concepts sont :

1) Le concept de la recherche et de la science;

2) Les domaines de la recherche ;

3) La question de départ;

4) l'exploration;

5) La construction de la problématique;

6) La collecte de données(les outils et la procédure);

7) L'analyse \& l'interprétation des données;

8) Le rapport de recherche (le contenu de chacune des parties d'un rapport de recherche).

Le résultat montre que les niveaux les plus dominants chez ces étudiants étaient entre: moyen et faible. Or, cette recherche essaie de constater l'efficacité du Weblogue sur le développement de quelques concepts de recherche en éducation chez l'échantillon déjà cité .Pour ce faire, on a tenté d'apporter une réponse aux questions suivantes:

1) Quels sont les concepts de recherche en éducation nécessaires aux étudiants de diplôme professionnel ,section du français, fac.de pédagogie?

2) Jusqu'à quel niveau ces étudiants possèdent -ils ces concepts?

\section{5}


3) De quoi se compose le blogue proposé? Quels sont ses contenus, ses tâches ainsi que ses outils?

4) Quelle est l'efficacité du blogue proposé sur le développement de quelques concepts de recherche en éducation nécessaires aux étudiants des études supérieures à la faculté de pédagogie?

\section{Hypothèses de la recherche:}

1) Il y a une différence statistiquement significative au niveau 0.01 entre la moyenne des notes des étudiants du groupe expérimental au pré-test et au post-test des concepts de recherche en sciences de l'éducation en faveur du post test;

2) Le blogue proposé a une grande efficacité sur le développement des concepts de recherche en sciences de l'éducation.

\section{Importance de la recherche (Quelles exploitations} pédagogiques sont possibles ?)

D'après Tomé $(2009,265)$ Plusieurs auteurs ont abordé les potentiels pédagogiques des weblogs en éducation. Nous résumons les plus importants.

- Les weblogs sont très faciles à utiliser et à intégrer dans différents contextes pédagogiques: interface intuitive, édition des textes et publication rapide sur le web. Ils sont ouverts au dialogue grâce à la fonction « Commentaires » (Deitering, Huston, 2004 ; Johnson, 2004 ; Man, 2004 ; Asselin, 2006).

- Les blogs représentent des outils dynamiques qui favorisent la création et la motivation chez les étudiants, ainsi qu'une communication authentique ; ils peuvent renforcer le travail collaboratif en classe (Downes, 2004 ; Stanley, 2005 ; Ward, 2004).

- Les weblogs permettent l'intégration d'activités et de tâches pédagogiques dans un espace réel de communication et le développement des projets de télécollaboration ou d'échanges entre classes (DemangeDucrot, 2006 ; Bernatchez, 2006; Tomé, 2007).

\section{6}


- L'utilisation des blogs en éducation modifie les rôles et les relations entre l'enseignant et les apprenants, en introduisant de nouvelles expériences pédagogiques pour la classe (O’Donnell, 2005 ; Lara, 2006 ; Tomé, 2007).

Campbell (2003) proposait une triple division des blogs dans l'enseignement des langues: tutor blog dans lequel l'enseignant organise les contenus du cours; learner blog, rédigé par un seul étudiant ou par un groupe d'étudiants; class blog, résultat de la collaboration entre la classe et l'enseignant. On peut donc résumer cette typologie des weblogs : le blog de l'enseignant, le blog de l'étudiant et le blog de la classe.

\section{Etapes de la recherche:}

\section{Notre travail s'est effectué en trois étapes:}

- La première étape concernant le cadre philosophique d' E-learninig et des blogues \& des concepts concernant la recherche en éducation .

- La deuxième étape consiste à;

1) Déterminer des concepts concernant la recherche en éducation nécessaires aux étudiants de études supérieures

2) Elaborer un test des concepts concernant la recherche en éducation et le blogue proposé, vérifier la validité des outils \& faire les modifications nécessaires;

3) Choisir un échantillon des étudiants de études supérieures, de diplôme professionnel, de section du français à la faculté de pédagogie de Ménoufya;

4) Appliquer le pré-test des concepts concernant la recherche en éducation aux sujets de l'échantillon;

5) Appliquer les tâches proposés à travers le blogue proposé aux sujets de l'échantillon;

6) Appliquer le post- test sur les sujets de l'échantillon.;

- La dernière est consacrée à analyser et à interpréter les résultats obtenus et à offrir quelques recommandations et d'autres sujets à étudier.

\section{7}




\section{PREMIEREMENT, CADRE PHILOSOPHIQUE.}

\section{A.La Recherche en science de l'éducation.}

\section{1-Qu'est-ce que " La science" ?}

Le mot science,tel qu'il est couramment utilisé,a au moins quatre significations différentes :il désigne une démarche intellectuelle visant à une compréhension rationnelle du monde naturel et social; il désigne un corpus de connaissances actuellement acceptées; il désigne la communauté des scientifiques,avec ses mœurs et sa structure sociale et économique; et, finalement, il désigne la science appliquée et la technologie. (Sokal,2013,2)

La science est une façon d'interroger et de connaître la réalité. Par rapport aux objets étudiés, elle réunit, dans un ensemble organisé, des connaissances vérifiées et vérifiables. Lamoureux ajoute que la réalité est vaste, car elle est composée de plusieurs phénomènes. Elle est également complexe, car chaque phénomène résulte de l'action combinée de différents éléments. La science pose un regard sur cette réalité, dans le but de la connaître. Ce regard a des particularités qui le distinguent de celui qui est posé par d'autres points de vue. L'essence de la science est des connaissances vérifiées et vérifiables. (Lamoureux, 2006,5). Le tableau (1) suivants présentent les objectifs de la science ainsi que les buts qui leur sont lies d'après Lamoureux(2006) .

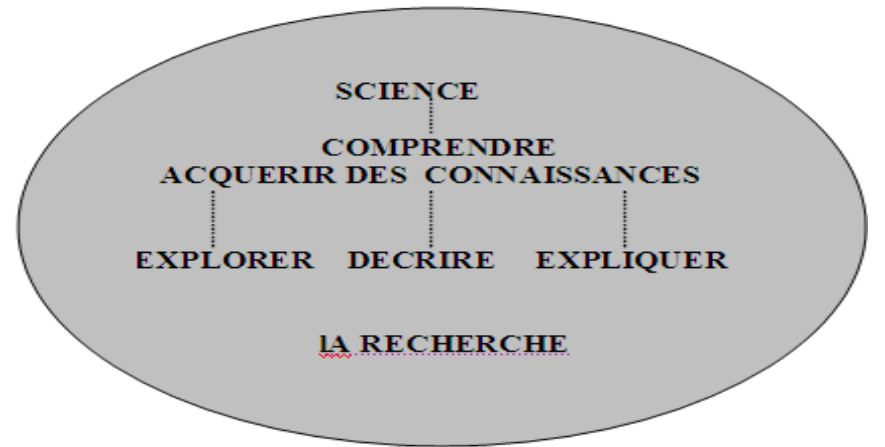

Figure (1) Les objectifs de la science (Lamoureux,2006,5)

\section{8}


Tableau (1) Les objectifs de la science d'après Lamoureux (2006,10)

\begin{tabular}{|lllc|}
\hline \multicolumn{2}{|c|}{ COMPRENDRE } & \multicolumn{1}{c|}{ CONTROLER } \\
ACQUERIR DES CONNAISSANCES & APPLIQUER DES CONNAISSANCE \\
Explorer & Décrie & Expliquer & Intervenir \\
-Identifier & Reconnaitre & Découvir & Modifier les éléments \\
-Nommer & les éléments & des relations & \\
-Comparer & Etablir des & de causalité & \\
-Classer & relations de & & \\
& Covariance & & \\
\hline
\end{tabular}

Comme il a été expliqué précédemment, la science veut saisir la réalité. Elle a donc comme objectif premier de la comprendre. Une fois cet objectif atteint, elle poursuit celui de contrôler la réalité. (Lamoureux, 2006,7)

\section{2-Qu'est-ce que "faire de la recherche" ?}

Pour répondre à cette question, il faut faire un détour (indispensable) sur ce qu'est la recherche, ses buts, ses traits.

La recherche est un cheminement vers une meilleure connaissance et elle doit être acceptée comme tel, avec tout ce que cela implique d'hésitations, d'errements et d'incertitudes. Dès lors, le chercheur doit s'obliger à choisir rapidement un premier fil conducteur aussi clair que possible de sorte que son travail puisse débuter sans retard et se structurer avec cohérence. Fondamentalement, toute recherche doit donc répondre à quelques principes stables et identiques, même si plusieurs voies différentes conduisent à la connaissance scientifique (Quivy\&Campenhoudt, 1995,,20-21). Trois traits fondamentaux de la recherche :

1) Elle vise à découvrir quelque chose nouvelle et non pas de répéter quelque chose. Autrement, dite Elle désigne une ouverture.

2) Elle vise une connaissance, il s'agit pour elle de connaître ou de savoir. Elle est donc liée à la vérité, elle est soucieuse de distinguer, parmi les représentations que l'on peut produire ou répéter,

\section{9}


celles qui répondent aux exigences d'une connaissance. (Initiation à la recherche en éducation,2007, François Coppens)

3) Elle est un travail, avec ce qu'il implique d'effort, de structuration, de continuité. Cette dynamique n'est pas immédiate, elle a une certaine durée, elle demande une résistance ou une capacité à surmonter les difficultés qui font partie inhérente de cette démarche.( Bachtold,2012,10)

De Ketele et Maroy définissent la recherche comme une «tentative d'objectivation du réel soumise à un certain contrôle empirique et social, ce qui suppose une procédure transparente et méthodique $»$. Le but d'une recherche scientifique est de faire progresser la connaissance d'une communauté sociale et d'augmenter les possibilités d'actions dont elle dispose pour modifier ou préserver l'existant. Les produits d'une telle entreprise sont donc les nouvelles connaissances produites à partir d'observations et d'actions sur le réel de référence de manière à " faire émerger des relations entre les phénomènes et donner du sens à des phénomènes particuliers » (De Ketele \& Maroy,2006, 222- 223).Une recherche est une entreprise car, selon ces auteurs, elle :

- procède en agissant sur le réel pris comme référence (le « réel de référence ») - non seulement regarder ou observer - en vue de réaliser un produit qui répond à un objectif spécifié ;

- suppose une organisation : de ressources humaines (ayant les compétences requises pour réaliser les objectifs poursuivis), des «objets » matériels ou immatériels sur lesquels on agit, des outils pertinents, des destinataires des produits construits (en l'occurrence dans la recherche scientifique: des connaissances produites) ;

- développe (et s'inscrit dans) une culture déterminée. (De Ketele\& Maroy,2006,224).

\section{0}


La recherche peut être résumée par une formule très simple:Posez une question, conduisez la recherche et répondez à la question. Une bonne recherche pose des questions claires et précises. La recherche doit utiliser des concepts et des définitions clairs. Et surtout, il est nécessaire d'utiliser des outils intellectuels appropriés (e.g. des cadres de travail, des méthodes et des technologies). En outre, une recherche est toujours menée en relation avec les recherches déjà réalisées sur le sujet et notamment la théorie. Le cadre théorique doit être basé sur les recherches (théories et résultats) antérieurs et les résultats de votre recherche doivent être débattus à la lumière de ces dernières. Enfin, différentes manières de présenter les résultats de la recherche sont acceptées. Le schéma : Qu'est-ce que la recherche? illustre ce principe (Class\&Schneider,2015)

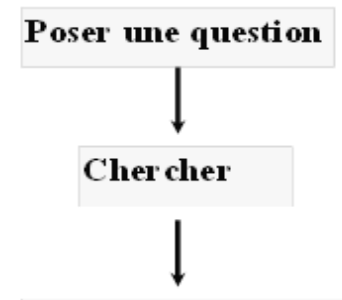

$$
\begin{aligned}
& \text { définir les limites de l'étude } \\
& \text { travailler les détails, affiner }
\end{aligned}
$$

utiliser des concepts et des définitions clairs

utiliser les outils adéquats

comparer à l'état de l'art (savoir existant)

Rép ondr e à la question avec un texte bien structuré (argumentation)

Figure (2). Notion de la recherche (Class\&Schneider,2015)

\section{Elle se caractérise par les principes de qualité suivants:}

\section{La recherche est une activité systématique:}

Les connaissances produites constituent un ensemble cohérent; Les résultats devraient s'intégrer à un système de connaissances (c'est-à-dire que vous les obtenez à partir de la littérature et que vous les comparez à la littérature)

\section{La recherche est centrée sur la réalité:}

par exemple la nature, la société, le comportement des gens, les attitudes des personnes,en d'autres termes: ne vous contentez pas de spéculer, observez les choses.

\section{La recherche utilise des outils précis:}

Ces outils sont les hypothèses, les théories, les méthodes, les techniques fiables, etc. Vous devriez également être

\section{1}

Journal of Arabic Studies in Education \& Psychology(ASEP) 
conscient de votre "biais de confirmation d'hypothèse" (tendance à privilégier les informations qui confirment votre hypothèse) et tester vos conclusions face à des explications alternatives.

\section{La recherche vise la généralisation:}

Elle contribue aux théories en utilisant (et en testant) leurs énoncés théoriques.Elle réutilise les instruments intellectuels (cadres de travail, grilles d'analyses, etc.). -Elle suggère des modifications des connaissances existantes (ou même de nouvelles théories).

\section{Une croyance dans le déterminisme:}

Les phénomènes sont les conséquences nécessaires de conditions (causes). En d'autres termes: le hasard dans les explications est uniquement dû à l'ignorance, à la complexité, etc.

\section{Un principe de relativisme:}

Nos connaissances ne sont pas parfaites, en particulier dans les sciences sociales où les humains sont à la fois sujets et objets, observateurs et observés, et où de nombreuses variables influencent un phénomène (Class\&Schneider,2015)

L'American Education Research Association [Association américaine pour la recherche sur l'éducation] (2006:34) suggère que la recherche peut contribuer à la connaissance des manières suivantes:

- Elle peut contribuer à une théorie déjà établie ou à une ligne de recherche empirique;

- Elle peut aider à établir une nouvelle théorie;

- Elle peut répondre à un besoin pratique ; ou

- Elle peut palier à un manque d'informations nécessaires sur un problème ou une question.

Il y a aussi une composante plus personnelle et sociale. Une recherche intéressante devrait tout d'abord vous

\section{2}

Journal of Arabic Studies in Education \& Psychology(ASEP) 
apporter de la satisfaction personnelle. Une recherche intéressante devrait également satisfaire une communauté donnée. Comme vous l'avez déjà découvert avec la rédaction de votre mémoire dans vos études antérieures, rédiger un travail de recherche académique est une entreprise d'envergure. Savoir que vous pouvez trouver la recherche intéressante et qu'elle peut se révéler utile pour votre carrière, par exemple, est un facteur fortement motivant qui vous aidera à la mener à bien. Bref,Elle est un déroulement structuré d'activités orientées vers la saisie de la réalité (Class\&Schneider,2015).

\section{Typologie de recherches en éducation}

Il se fait de la recherche dans deux domaines différents. Dans le domaine fondamental, il s'agit d'augmenter la somme des connaissances scientifiques, sans égard à leurs applications immédiates possibles; dans le domaine appliqué, il s'agit plutôt de trouver un savoir qui servira immédiatement dans une situation concrète. Ces deux domaines sont complémentaires. (Karsenti\&Zajc,2000, 101).

Le tableau 2 résume les principales caractéristiques de ces deux domaines.

Tableau (2) Les caractéristiques des domaines de la recherche fondamentale et de la recherche appliquée.(Karsenti\&Zajc,2000,101)

\begin{tabular}{|c|c|c|}
\hline \multicolumn{2}{|c|}{ Recherche fondamentale } & Recherche appliquée \\
\hline $\begin{array}{l}\text { Point de } \\
\text { départ }\end{array}$ & $\begin{array}{c}\text { Préoccupations théoriques } \\
\text { Connaissances déjà établies } \\
\text { (hypothèse) }\end{array}$ & $\begin{array}{c}\text { Préoccupations concrètes } \\
\text { Situation problème (plan } \\
\text { d'action) }\end{array}$ \\
\hline But & $\begin{array}{l}\text { Augmenter la somme des } \\
\text { connaissances }\end{array}$ & $\begin{array}{c}\text { Permettre la prise de } \\
\text { décisions éclairées }\end{array}$ \\
\hline Résultats & $\begin{array}{c}\text { Connaissances généralisables } \\
\text { Niveau d'abstraction } \\
\text { Nouveau savoir théorique }\end{array}$ & $\begin{array}{l}\text { Connaissances spécifiques } \\
\text { Niveau de spécialisation } \\
\text { Nouveau savoir pratique }\end{array}$ \\
\hline
\end{tabular}

\section{Les règles d'éthique en recherche.}

Selon Demers (1994,83-84) les règles d'éthique en recherche sont un ensemble des règles morales présentées 
sous forme de lois que le chercheur doit respecter. Tout chercheur, quel que soit son niveau, doit s'engager à respecter les règles morales . Ces règles ont pour but non seulement de protéger les participants d'une recherche, mais également de protéger la société contre des manipulations ou des abus qui pourraient être commis par certains chercheurs. Toute personne a donc avantage à les connaître. Dès qu'il conçoit un projet de recherche, le chercheur doit avoir des préoccupations d'ordre éthique. Ces préoccupations se résument dans les quatre points suivants:

Elaborer un projet de qualité. Le chercheur a la responsabilité d'élaborer le meilleur projet de recherche possible. Pour cela, il doit, entre autre choses, s'assurer que sa recherche constitue un avancement en regard des connaissances scientifiques actuelles. De plus, il ne doit pas accepter des commentaires qui diffuseraient de façon restreinte et partiale les résultats obtenus.

Se préoccuper des enjeux. Dès la conception de son projet, le chercheur doit se préoccuper des enjeux possibles de sa recherche. Ainsi, il ne peut uniquement satisfaire sa propre curiosité: les résultats et les conclusions de sa recherche doivent avoir une certaine importance pour la société ou pour certains de ses membres. De plus, le chercheur doit envisager les conséquences négatives possibles de ses découvertes sur les individus et sur la société.

Peser les coûts sociaux. Le chercheur se trouve devant deux droits: celui qu' a la société d'augmenter son savoir, et celui qu'a le participant de voir sa dignité et ses droits individuels respectés. Il a donc la responsabilité de peser les coûts sociaux de sa recherche. Pour cela, il doit en prévoir les avantages théoriques et pratiques pour la société et les inconvénients qu'elle risque d'entraîner pour les

\section{4}

Journal of Arabic Studies in Education \& Psychology(ASEP) 
participants. L'évaluation des avantages et des inconvénients n'a pas trait seulement à leur nature, mais également à leur degré et à leur caractère permanent.

Assurer la rigueur méthodologique de sa recherche. Pour assurer la valeur des résultats obtenus, le chercheur doit respecter un certain nombre de normes.

- Eviter toute forme de discrimination dans la sélection des participants;

- Traiter de façon complète et objective les données obtenues: il ne doit ni omettre ni camoufler celles qui ne concordent pas avec son hypothèse;

- Reconnaître la portée limitée de ses interprétations et veiller à ce qu'elles ne soient pas modifiées par des gens qui y trouveraient un intérêt.

- Publier ses résultats, pour les rendre accessibles à la communauté scientifique et les soumettre à la critique de ses pairs.

- Respecter les droit d'auteur et de propriété intellectuelle: il ne doit pas "emprunter" les mots ou les idées d'un autre sans lui en accorder le crédit.

\section{Les étapes de la démarche cientifique.}

Une démarche est une manière de progresser vers un but. Exposer la démarche scientifique consiste donc à décrire les principes fondamentaux à mettre en œuvre dans tout travail de recherche. Bachelard (1965) a résumé la démarche scientifique en quelques mots:

\section{"le fait scientifique est conquis, construit et constaté",}

- conquis sur les préjugés;

- construit par la raison;

- constaté dans les faits.

Bourdieu \& autres (1968) \& Berthelot(1990,39) décrivent la démarche comme processus en trois actes dont l'ordre doit être respecté. Ces trois actes sont :

\section{5}




\section{La rupture}

La rupture consiste précisément à rompre avec les préjugés et les fausses évidences qui nous donnent seulement l'illusion de comprendre les choses. La rupture est donc le premier acte constitutif de la démarche scientifique.

\section{La construction}

Cette rupture ne peut être effectuée qu'en se referant à un système conceptuel organisé, susceptible d'exprimer la logique que le chercheur suppose être à la base du phénomène. C'est grâce à cette théorie qu'il peut construire des propositions explicatives du phénomène à l'étude et qu'il peut revoir le plan de recherche à installer, les opérations à mettre en œuvre et les conséquences auxquelles il faut logiquement s'attendre au terme de l'observation. Sans cette construction théorique, il n'y aurait pas d'expérimentation valable. Il ne peut $y$ avoir, en sciences sociales, de constatation fructueuse sans construction d'un cadre théorique de référence.

\section{La constatation}

Une proposition n'a droit au statut scientifique que dans la mesure ou elle est susceptible d'être vérifiée par des informations sur la réalité concrète. Cette mise à l'épreuve des faits est appelée constatation ou expérimentation. Elle correspond au troisième acte de la démarche.

\section{6-Les étapes de la démarche.}

Les trois actes ne sont pas indépendants les uns des autres. Ils se constituent au contraire mutuellement. Ainsi par exemple, la rupture ne se réalise pas uniquement en début de recherche; elle s'achève dans et par la construction. En revenche, celle-ci ne peut se passer Dans le déroulement concret d'une recherche, les trois actes de la démarche scientifique sont réalisés au cours d'une succession d'opérations qui sont regroupées en sept étapes (Campenhoudt\&Quivy,1995,17-18) . Le schéma suivant

\section{6}


distingue de manière précise les étapes les unes des autres (Karsenti\& Savoie-Zajc,2000,129):

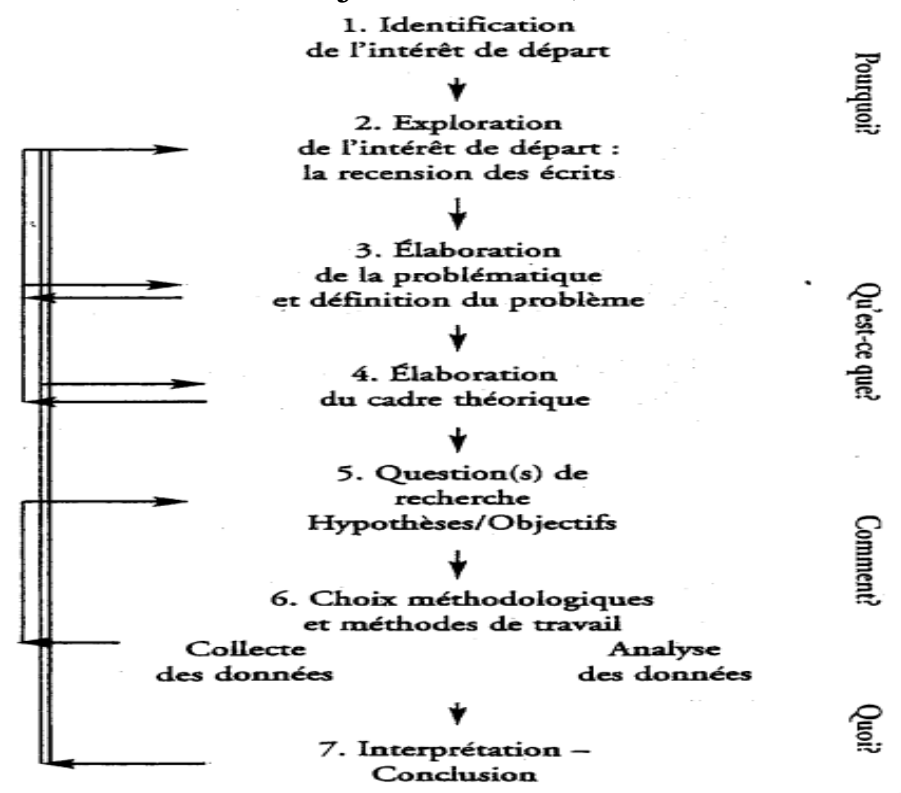

Figure(3).Les étapes de la démarche scientifique en recherche en éducation (Karsenti \&Savoie-Zajc, 2000,129).

\section{1-La question de départ}

D'après Quivy\&Campenhoudt(1995,35) la meilleure manière d'entamer un travail de recherche consiste à s'efforcer d'énoncer le projet sous la forme d'une question de départ. Par cette question, le chercheur tente d'exprimer le plus exactement possible ce qu'il cherche à savoir, à élucider, à mieux comprendre. La question de départ servira de premier fil conducteur à la recherche. Son rôle consiste à «traduire ce qui se présente couramment comme un centre d'intérêt ou une préoccupation relativement vague en un projet de recherche opérationnel ».

Pour remplir correctement sa fonction, la question de départ doit présenter des qualités de clarté, de faisabilité et de pertinence:

1) La clarté : Qu'elle soit concise, sans aucun de ces entortillements qui font que finalement on ne sait plus ce que l'on vise; qu'elle soit précise,

\section{7}

Journal of Arabic Studies in Education \& Psychology(ASEP) 
c'est-à-dire qu'elle définisse un champ de réalité bien circonscrit, et non pas vague ou potentiellement infini ; et enfin qu'elle soit univoque, que chacun qui entend cette question comprenne bien ce qu'elle vise, qu'il n'y ait pas de mot ou d'expression qui soit équivoque, qui puisse avoir plusieurs sens sans que l'on sache clairement lequel était visé.

2) La faisabilité : Une question de départ doit définir une tâche qui correspond aux moyens que l'on a. Le chercheur doit toujours se demander si la question est réaliste, si la tâche qu'elle définit correspond à un champ que l'on est capable de couvrir avec les moyens (temps, finances, compétences...) dont on dispose.

3) La pertinence : Pour être pertinente, une question de départ doit être une vraie question, et non pas une affirmation ou une opinion à laquelle on ajoutera un point d'interrogation pour faire semblant qu'il s'agit d'une question. Elle doit aussi orienter vers les réalités, vers la réalité, et non pas vers des spéculations, des fantasmes, des spéculations sur l'avenir: elle doit provoquer une confrontation et un échange avec d'autres, autour ou à propos d'une réalité à laquelle ils peuvent avoir accès. Elle doit aussi relever du champ d'études auquel la recherche, le cas échéant, est censée appartenir.

\section{2-L'exploration}

Le projet de recherche ayant été provisoirement formulé sous la forme d'une question de départ, il s'agit ensuite d'atteindre une certaine qualité d'information sur l'objet étudié et de trouver les meilleures manières de l'aborder. C'est le rôle du travail exploratoire. Celui-ci se compose de deux parties qui sont souvent menées parallèlement: d'une part un travail de lecture et d'autre part des entretiens ou d'autres méthodes appropriées .Au terme de cette étape, le chercheur peut être amené à reformuler sa question de

\section{8}


départ d'une manière qui tienne compte des enseignements de son travail exploratoire..Le but est de préciser la question de départ et de la reformuler. Il est aussi de faire "l'état de l'art" sur le thème considéré(Quivy,R \& Campenhoudt,1995,81).

\section{3-La problématique.}

La meilleure définition de ce qu'est un problème scientifique (la fameuse question de recherche qui doit orienter le travail) est la plus simple :

[...] la connaissance commence par la tension entre savoir et non-savoir : pas de problème sans savoir - pas de problème sans non-savoir. Un problème scientifique a la forme d'une tension entre savoir et non-savoir. (Popper, 1979, 76 ; cité par Dumez, 2010, p. 9).

La problématique est l'approche ou la perspective théorique qu'on décide d'adopter pour traiter le problème posé par la question de départ. Elle est une manière d'interroger les phénomènes étudiés. Elle constitue une étape charnière de la recherche, entre la rupture et la construction.La formulation d'une problématique constitue dans la dynamique de la recherche un moment qui est à la fois décisif et difficile. (Brent, 2005, 399).

Après avoir formulé une question de départ, après avoir mené une exploration dans le champ ouvert par cette question de départ, le chercheur rencontre différentes problématiques ou différentes approches possibles de l'objet qui l'intéresse et sur lequel porte sa question de départ. Il doit, à ce moment, choisir l'angle qui lui semble le plus intéressant et le plus pertinent parmi ceux qu'il a rencontrés. Il doit choisir l'une des problématiques, l'une des approches ou l'un des aspects, qui peuvent correspondre à l'objet qui l'intéresse, dans le vaste champ des recherches et des études ouvert par sa question de départ. Pour formuler une problématique, il convient de

1) Mettre à plat les différentes problématiques qui ont été rencontrées dans le champ ouvert par la

\section{9}


question de départ et qui sont des manières légitimes d'approcher la réalité interrogée.

2) Choisir, parmi ces approches possibles, celle qui semble la plus intéressante et pertinente, à la fois pour le chercheur lui-même, pour l'objet étudié et éventuellement quant à l'apport qu'elle permettra pour l'ensemble de la communauté de recherche.( Quivy \& Campenhoudt,1995,102).

Angeline (2007)explique une problématique dans le cadre d'une étude ou d'un rapport comme:

- Un processus : problématiser un sujet c'est le questionner pour déterminer la façon la plus appropriée de l'étudier.

- Un produit : la problématique est la synthèse de ce questionnement qui est présentée en introduction du rapport ou de la note. Elle annonce le sujet de la commande, argumente les choix faits et annonce comment elle sera traitée, en termes de méthodes d'enquête comme d'options prises.
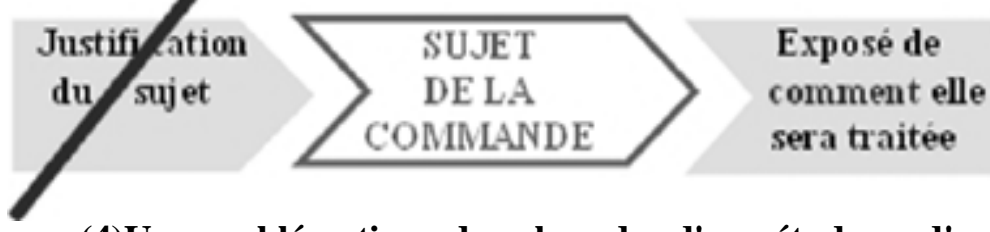

Figure (4)Une problématique dans le cadre d'une étude ou d'un rapport (Angeline, 2007)

La problématisation est un processus qui permet de passer d'un thème de recherche général, ou d'une question de départ classiquement qualifiée de naïve, à des hypothèses de travail précises. Elle procède par étapes successives d'exploration des possibles (cadres théoriques, aspects de la question, hypothèses, etc.) et de choix d'options pour progressivement délimiter le travail à entreprendre.

\section{La revue de littérature\&Le Cadre conceptuel}

Un domaine de recherche renvoie souvent à une discipline. Une recherche peut avoir une orientation

\section{0}

Journal of Arabic Studies in Education \& Psychology(ASEP) 
psychologique, sociologique, économique, historique, pédagogique, didactique, etc. D'où l'utilité, en fonction de l'envergure et de l'objectif de la recherche entreprise, de consulter les travaux les plus récents dans le domaine ou dans des domaines connexes.Le principal objectif de la revue critique de la littérature est de situer, d'insérer, d'affilier une recherche dans un courant théorique élaboré par d'autres recherches et de prendre position de manière critique par rapport à leurs conclusions (Sall , 2016,11).

Ce que l'on attend d'un travail de recherche est l'originalité. L'originalité est tout le sens de la revue de littérature. Il faut bien maîtriser ce qui a déjà été fait en matière de recherche pour pouvoir positionner sa propre recherche de manière à ce qu'elle apporte quelque chose de plus, à ce qu'elle soit originale (à la frontière du savoir et du non-savoir, comme on l'a vu). (Dumez,, 2011,18) :

C'est l'objet même de la revue de littérature que de préciser la sphère du déjà fait et déjà connu, et d'identifier les frontières de la connaissance pour déterminer une question de recherche originale. D'où les objectifs de la revue de littérature (Hart, 2009, p. 27) :

- identifier la frontière entre ce qui a déjà été fait et qui a besoin d'être étudié;

- découvrir des variables importantes liées au sujet ;

- faire une synthèse et élaborer une perspective nouvelle ;

- identifier des relations entre des idées et des pratiques ;

- établir le contexte du problème ;

- établir la signification du problème ;

- acquérir le vocabulaire et les concepts liés au problème ;

- comprendre la structure du sujet ;

- établir un lien entre les idées et les cadres théoriques d'une part, et leurs applications de l'autre ;

- identifier les méthodes et techniques de recherche qui ont déjà été utilisées pour traiter du problème ;

\section{1}


- replacer le sujet dans une perspective historique de manière à montrer que l'on maîtrise à la fois l'histoire du problème et l'état le plus récent de son développement.

Face à une telle complexité des dimensions possibles de l'originalité et des objectifs de la revue de littérature qui y sont liés, quelles formes \&comment procéder?

\subsection{Formes typiques de revue de littérature}

Cooper (2010) cité par Creswell $(2014,28)$ identifie quatre formes différentes de revue de littérature:

- Une intégration des travaux des autres chercheurs en les organisant de manière thématique et en mettant en avant les problèmes principaux;

- Une critique des travaux académiques antérieurs;

- Un pont entre différents sujets reliés;

- Une identification de questions et problèmes centraux dans un domaine donné.

En effet, il est important de bien identifier la fiabilité de ses sources avant de se baser dessus (e.g. un article trouvé sur internet peut être intéressant mais s'il n'a pas été publié, il est plus sage de le considérer comme du matériel secondaire) (Creswell ,2014, 29).

\subsection{Réaliser une carte de la littérature}

La carte de la littérature est un artefact visuel qui synthétise les recherches conduites par d'autres chercheurs sur le sujet. Pour réaliser cette carte, qui peut prendre des formes multiples, les règles de conceptions utilisées pour l'élaboration de cartes conceptuelles sont applicables: (Novak \& Cañas, 2008,22).

1) Élaborez d'abord une question à laquelle le réseau de concepts tentera de répondre (concept central).

2) Recenser d'abord les concepts clefs les plus généraux (en surface) associés à la question de départ. Ensuite, établissez la liste des concepts plus spécifiques (en profondeur).

\section{2}

Journal of Arabic Studies in Education \& Psychology(ASEP) 
3) Regroupez et hiérarchisez les concepts selon l'organisation naturelle de ceux-ci.

4) Reliez les concepts entre eux pour former des propositions (liens proches et liens éloignés). Si vous souhaitez proposer au lecteur un ordre de lecture de votre réseau de concepts, n'hésitez pas à numéroter les concepts et/ou les relations qui les unissent.

5) Ajouter des ressources au besoin (URL, vidéo, images, textes, etc.) ou des exemples.

6) Réviser votre travail (ajout de sous concepts et de relations, couleur, police).

7) Partager votre réseau de concepts

\subsection{Réaliser un tableau de littérature}

Il s'agit alors de résumer les concepts les plus importants que vous trouvez dans la littérature et d'identifier des relations implicites (quels concepts sont associés à quels auteurs). Cette technique est basee sur un tableau à double entrée: dans les colonnes, vous mettez les concepts et dans chaque ligne un article.

Tableau (3).Tableau de littérature (SALL,2016)

\begin{tabular}{|c|c|c|c|c|c|}
\hline & \multicolumn{5}{|c|}{ Concepts } \\
\hline Articles & Concept A & Concept B & Concept C & Concept D & $\ldots$. \\
\hline 1 & & $\mathrm{X}$ & $\mathrm{X}$ & & \\
\hline 2 & $\mathrm{X}$ & & $\mathrm{X}$ & $\mathrm{X}$ & \\
\hline$\ldots$ & & & & & $\mathrm{X}$ \\
\hline
\end{tabular}

\subsection{Les résumés}

Dans le même temps, il est important, pour vous, de faire un résumé de chaque étude/article en spécifiant:

- Le problème étudié;

- L'objectif principal de l'étude;

- La méthodologie utilisée, y compris brièvement l'échantillon, etc.

- Les résultats clés;

\section{5.. Elaboration du cadre théorique.}

On appelle, cadre théorique, l'ensemble des connaissances, théories, qui ont un rapport quelconque avec

\section{3}

Journal of Arabic Studies in Education \& Psychology(ASEP) 
le sujet de la recherche.Les cadres théoriques fournissent un aperçu du phénomène (éléments et relations) et permettent de combler l'écart entre la théorie et la recherche empirique .Ils facilitent l'analyse et la formulation de questions de recherche (par exemple les causalités à étudier, les éléments utiles) $\mathrm{Du}$ reste,Ils aident à trouver des instruments empiriques pour mesurer un concept (SALL,2016).

Cette partie de la recherche est longue, elle porte en ellemême ses bénéfices.Elle ouvre des horizons, elle dévoile des aspects inédits. Elle permet au chercheur d'apprivoiser son sujet et de s'en imprégner.Il doit être convaincu qu'avant d'apporter sa contribution à la progression du savoir,il est nécessaire qu'il s'enrichisse des connaissances qui entourent son travail.

\section{5-Question (s) de recherche}

\section{1-Pourquoi des hypothèses?}

Durkheim (1991), Quivy et Campenhoudt (1995,117119) affirment que l'organisation d'une recherche autour d'hypothèses de travail constitue le meilleur moyen de la mener avec ordre et rigueur sans sacrifier pour autant l'esprit de découverte et de curiosité propre à tout effort intellectuel digne de ce nom. Bien plus, un travail ne peut être considéré comme une véritable recherche s'il ne se structure autour d'une ou de plusieurs hypothèses. Pourquoi?

D'abord parce que l'hypothèse traduit par définition cet esprit de découverte qui caractérise tout travail scientifique. Fondée sur une réflexion théorique et sur une connaissance préparatoire du phénomène étudié (phase exploratoire), , elle se présente comme une présomption non gratuit portant sur le comportement des objets réels étudiés.

Mais en même temps, l'hypothèse procure à la recherche un fil conducteur particulièrement efficace qui, à partir du moment où elle est formulée,remplace la question de

\section{4}

Journal of Arabic Studies in Education \& Psychology(ASEP) 
recherche dans cette fonction, même si celle-ci doit rester présente à l'esprit. La suite du travail consistera en effet à tester les hypothèses en les confrontant à des données $d$ d'observation.

Sous les formes et les procédures les plus variées, les recherches se présentent toujours comme des va-et-vient entre une réflexion théorique et un travail empirique. Les hypothèses constituent les charnières de ce mouvement; elles lui donnent son amplitude et assurent la cohérence entre les parties du travail.

\section{2-Qu'est-ce qu'une hypothèse ?}

Une hypothèse est une prédiction des résultats à venir. Habituellement, elle prédit une relation entre deux ou plusieurs événement, qui peuvent être:

deux faits (ex: les étudiants qui échouent à un cours augment leur temps d'étude hebdomadaire); *un fait et un concept( ex: à la suite d'un échec scolaire, l'estime de soi des étudiants baisse); *deux concepts(ex: à mesure que l'estime des soi des étudiants baisse, leur confiance dans l'avenir baisse également).

Une hypothèse peut prédire deux sortes de relation :**un lien de convariance: elle prédit que des changements surviennent dans l'événement $\mathrm{Y}$ en même temps que des changements dans l'événement $\mathrm{X}$.

un lien de causalité : elle prédit que tel changement dans l'événement $\mathrm{X}$ produit tel changement dans l'événement $\mathrm{Y}$ .(Lamoureux,2006,111).

\section{3-Un choix}

Selon le but général de la recherche, la méthode employée et les informations relevées dans la recension des écrits, il est donc approprié soit poser un objectif, soit d'énoncer une hypothèse. La figure 4.. montre ces différentes possibilités(Lamoureux,2006,112).

\section{5}




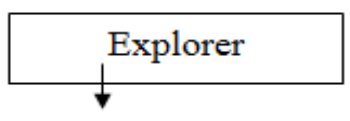

Objectif

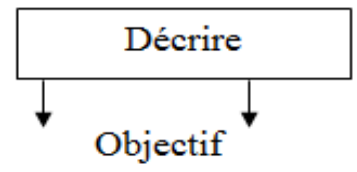

$$
\begin{array}{ccc}
\text { Méthode } & \text { Méthode d'analyse de contenu } & \\
\cline { 2 - 3 } \text { historique } & \text { Méthode d'enquête } & - \\
\text { Méthode } & \text { Méthode } \\
\text { corrélationnelle } & \begin{array}{c}
\text { expérimentale } \\
\text { nand }
\end{array}
\end{array}
$$

Hypothèse (lien de causalité)

Figure(5)Le lien entre les buts de la recherche, la méthode employée et l'énoncé d'un objectif ou d'une hypothèse.

\section{4-Les six caractéristiques d'une hypothèse}

L'hypothèse d'une recherche ont en commun six caractéristiques. Les deux premières concernent particulièrement la façon d'en rédiger l'énoncé. (Lamoureux ,2006, 113)

\section{1-L'affirmation}

L'énoncé d'un hypothèse d'une recherche doit être formulé comme une affirmation, non comme une question . De lus, on le rédige au présent, et non au futur.

\section{2-L'efficacité}

L'énoncé d'une hypothèse doit être concis, tout en étant très explicite. Il exprime la manière concrète par laquelle il sera confronté aux faits.

\section{3- La justification}

Le contenu d'une hypothèse doit être cohérent avec les connaissances actuelles relevées dans la recension des écrits. C'est pourquoi on ne dit jamais qu'il est vrai ou faux; on dit qu'il est justifié ou non justifié. L'énoncé d'une hypothèse découle directement du raisonnement logique du chercheur tout au long de la construction de sa problématique.

\section{4-La précision}

Une hypothèse doit être aussi précis . L'énoncé d'une hypothèse contient donc les éléments concrets (observables 
et mesurables) qui renseignent le lecteur sur l'action que le chercheur entreprendra pour interroger des faits de la réalité. La formulation d'une hypothèse doit également être précise dans sa prédiction d'une relation entre deux événements, qu'elle prédise un lien de covariance ou un lien de causalité.

\section{$\underline{\text { 5- La vérifiabilité }}$}

Les hypothèses doivent être vérifiables; ils doivent pouvoir être soumis directement à l'épreuve des faits recueillis dans la réalité. Au terme de la collecte et de l'analyse des données, le chercheur sera ainsi en mesure d'apporter une réponse à sa question de recherche.

\section{La productivité}

Un objectif et une hypothèse doivent être productifs sur le plan théorique; dans la somme des connaissances déjà accumulées, ils apportent un élément nouveau, . C'est le deuxième mouvement de la relation circulaire entre la réalité théorique et la réalité empirique

\section{6- Choix méthodologique}

\subsection{La collecte des données.}

Cette opération consiste à recueillir ou rassembler concrètement les informations prescrites auprès des personnes ou unités d'observation retenues dans l'échantillon(Quivy \& Campenhoudt, 1995 ,184).

D'autre part Livian $(2015,33)$ distingue habituellement les "données primaires" (celles que le chercheur a obtenues en direct) et les "données secondaires" (celles provenant de sources existantes).Les deux sont nécessaires mais la dose de données secondaires doit être limitée, car sinon on considérera la recherche comme peu originale, "de seconde main". D'une manière ou d'une autre, le chercheur doit "construire" ses propres données, c'est-à-dire choisir les faits ou les phénomènes à observer, les saisir, les mesurer (s'il y a lieu) et les traiter. La recherche doit avoir une "base empirique" (répondre à la question : quelle réalité a été observée ?).

\section{7}

Journal of Arabic Studies in Education \& Psychology(ASEP) 
IL ne suffit pas de concevoir un bon instrument, il faut encore le mettre en œuvre de manière à obtenir un taux de réponses suffisant pour que l'analyse soit valable. Bref, il importe que le chercheur ait une vision globale de son travail et ne prévoie les modalités d'aucune de ces étapes sans s'interroger constamment sur ses implications ultérieures. On affirme qu' il existe une interdépendance entre l'observation et l'analyse des données. Il faut donc anticiper et se demander régulièrement pour chaque réponse prévue: " Est-ce que la question que je pose va me donner l'information et le degré de précision dont j'ai besoin dans la phase ultérieure?" ou encore:" A quoi doit servir cette information et comment vais-je pouvoir la mesurer et la mettre en relation avec les autres?".Un panorama sera présenté, qui portera quant à lui sur les méthodes de collecte et d'analyse des informations ( Quivy \& Campenhoudt,1995 ,187).

\subsection{L'analyse des données}

Les données sont traitées, analysées d'une manière que l'étudiant devra indiquer. Ce traitement sera la conséquence du choix méthodologique réalisé. Qu'il s'agisse d'un traitement de données quantitatives (des chiffres) ou qualitatives (des discours, des textes), le chercheur aura recours à des méthodes connues ou des logiciels qu'il devra indiquer. L'analyse de données aura comme conclusion soit la vérification des hypothèses posées au départ (ou leur invalidation), soit une interprétation globale fournissant une réponse à tout ou partie des questions de recherche posées au départ.(Livian,2015,33).

L'analyse des données est l'étape qui traite l'information obtenue pour la présenter de manière à pouvoir comparer les résultats observés aux résultats attendus par hypothèse. Dans le scénario d'une analyse des données quantitatives, cette étape comprend trois opérations. Toutefois, les principes de la démarche peuvent, en grande parti, être transposés à d'autres types de méthodes.

\section{8}


1) La première opération consiste à décrire les données. Cela revient, d'une part, à les présenter sous la formes requise par les variables impliquées dans les hypothèses et, d'autre part, à les présenter de manière à ce que les caractéristique de ces variables soient bien mises en évidence par la description.

2) La deuxième opération consiste à mesurer les relations entre les variable, conformément à la manière dont ces relations ont été prévues par les hypothèses

3) La troisième opération consiste à comparer les relations observées aux relations théoriquement attendues par hypothèse et à mesurer l'écart entre les deux. Si l'écart est nul ou très faible, on pourra conclure que l'hypothèse est confirmée; sinon il faudra examiner d'où vient l'écart et tirer des conclusions appropriées.(Quivy \& Campenhoudt, 1995,243).

\section{Conclusion}

Il s'agit du moment de synthétisation de l'ensemble du travail, depuis la problématique jusqu'à la discussion. La Conclusion met en valeur les points saillants du travail et signale les limites de la recherche (aucune idée nouvelle, aucun concept ne sont attendus au niveau de la conclusion) tout en proposant des perspectives.(Ministère de l'Education au Liban,2015,11).

\section{B. Les WebBlogs}

\section{Introduction}

L'essor des nouvelles technologies de l'information et de la communication a favorisé de nombreuses applications dans le monde de l'éducation.Depuis le grand succès connu par les blogs parmi les internautes (journalistes, informatique, journal personnel) les enseignants ont essayé de les intégrer dans leurs pratiques éducatives.Le blog

\section{9}


constitue 1`aaa un mode alternatif d'expression pour les étudiants et en même temps un mode de réflexion sur ce qu'ils apprennent et sur la façon dont ils apprennent .Du reste, L'intégration des blogs dans la classe transforme la relation pédagogique entre l'enseignant et les étudiants, car ceux-ci peuvent s'exprimer en tant qu'auteurs et construire des connaissances par eux-mêmes ,et favorise ainsi les pédagogies actives et socio-constructivistes (Tomé,2007,341).

"Jusqu'à ces dernières années,on parlait de site,de portail,de pages Web. Mais personnene connaissait le mot «blog».Aujourd'hui,les blogs fleurissent. On en Compterait entre 5 et 10 millions dans le monde. Il est désormais raisonnable de parler de «phénomène des blogs», avec un impact direct sur le volume d'informations disponibles. Les blogs, c'est le Web en temps réel, le Web vivant,celui qui secrée perpétuellement."( Paoli,2006,91)

\section{Qu'est-ce qu'un blog?}

Un «blog» (en français blogue, journal web ou carnet web) est un site internet personnel permettant à un internaute de publier facilement des informations en ligne sous forme de journal. Le terme «blog» est la contraction de «weblog» (au même titre, «blogue» est la contraction de «weblogue»). Le blogest donc une pageweb,s'appuyant généralement sur un outil dynamique permettant de gérer la mise en page du contenu, dans la quelle son auteur publie des actualités,des liens vers des sites web ou de courts textes de sa création.( Paoli,2006,91)

Le site Pointblog.com16 fut l'un des premiers à définir de manière simple et consensuelle ce qu'est un blog: «De web - toile - et log - carnet de bord -,un blog est un site web (...) composé essentiellement d'actualités, publiées au fil de l'eau et apparaissant selon un ordre ante-chronologique, le

\section{0}


plus souvent enrichies de liens externes et de commentaires.» (Le site Pointblog.com16,2011)

Un blog ou carnet web est un site web organisé chronologiquement et actualisé périodiquement (calendrier, archives, le dernier message s'affiche en première position) qui s'appuie sur une technologie de type CMS (système de gestion de contenu). Son interface d'édition simplifiée et sa facilité d'utilisation permettent son emploi en classe de français langue étrangère (FLE) grâce à la publication immédiate de pages web et à la réalisation de différentes tâches pédagogiques.(Tomé,2007,341)

Le blog ou weblog désigne un outil de publication personnelle sur Internet. "De façon très synthétique, un blog est un type de site composé essentiellement d'actualité (ou billets) publiés au fil de l'eau et apparaissant selon un ordre chronologique (les plus récents en haut de page), le plus souvent enrichies de liens hypertextes externes »(Fievet \& Turrettini. 2004)

Les weblogs sont, donc, à première vue de simples sites personnels écrits par une ou plusieurs personnes. Cependant, leur grande nouveauté réside dans le fait qu'ils sont très faciles à créer et surtout à mettre à jour. Ils permettent, ainsi, à tout internaute de s'exprimer sur Internet, leur utilisation ne nécessitant aucune compétence technique, ce qui explique leur immense succès.( Garreau,2005,4)

Ainsi, selon Benoit Desavoye "le phénomène blog accompagne la croissance fulgurante de l'utilisation d'Internet, sa vulgarisation et son intégration à nos habitudes quotidiennes ». (Desavoye\&Ducamp et al ,2005)

La figure suivante nous montre l'apparence générale d'un blog sur Blog.france2.fr . 


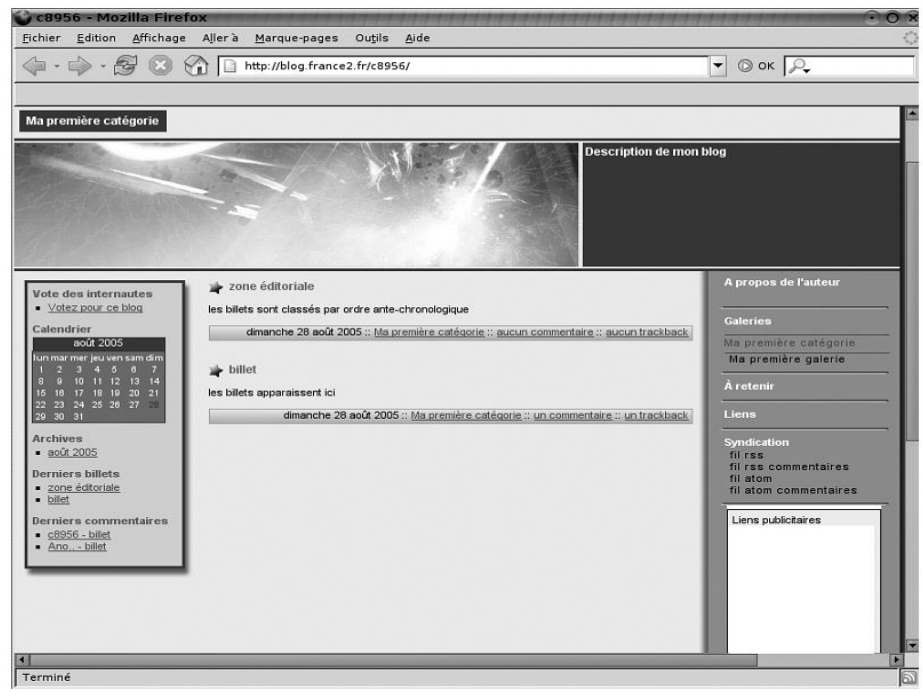

Figure (6) L'apparence générale d'un blog

Un weblog est une page web dynamique. Bien que certaines constantes formelles se retrouvent d'un blog à l'autre, il n'existe pas une présentation spécifique aux blogs.Le blog se compose d'une liste d'articles, appelés aussi billets ou notes, rangés par date dans la zone éditoriale.Les billets sont généralement classés par ordre chronologique inverse, c'est-à-dire que le plus récentapparaît en haut de liste.Les colonnes encadrant la zone éditoriale comprennent des blocs, ou modules, annexes: calendrier, archives, blogroll, rubriques, etc. Les hauts et bas de page peuvent être occupés par des blocs, par des éléments graphiques (logo, photos, etc.), ou encore par $\mathrm{du}$ texte. Le weblog intègre les dernières possibilités multimédias de l'Internet. Il contient aussi bien du texte, que des images, du son et de la vidéo, tous au format numérique. Enfin, il est ouvert sur le reste du Web avec de nombreux liens hypertextes venant étayer ou documenter les propos.( Ertzscheid,2011, $7 \leqslant$ ).

\section{3-Ses caractéristiques}

Desavoye et Ducamp (2005)propose six grandes caractéristiques propres aux blogs :

\section{2}


1) Tout d'abord un blog se compose d'une liste de billets (ou post) classés chronologiquement (du plus récent au plus ancien), constituant la «colonne vertébrale » du blog. Pour chaque billet, la date de publication est mentionnée ainsi que le nombre de commentaires faits sur le post, en cliquant sur ce nombre on accède directement aux commentaires. En effet, chaque entrée d'un blog peut être commentée par le visiteur qui le souhaite, de façon anonyme ou non. De plus, à chaque billet est associée une ancre, le permalink. Ce lien permanant permet de retrouver instantanément un message déterminé. Par conséquent, il devient aisé de faire référence à un message bien précis posté sur un autre weblog. En effet, l'autre caractéristique du blog est le trackback (aussi appelé rétrolien ou pisteur). Il consiste à faire apparaître, sur un blog, le titre, le lien permanent, et un extrait du texte écrit sur un autre blog, par une autre personne. Ce système très simple de citation favorise, alors, le respect du droit d'auteur, la manipulation très simple permettant de mettre en valeur la source originale.

2) L'autre caractéristique du blog est l'archivage. Tous les billets et commentaires sont automatiquement archivés selon le délai choisi par le blogueur. Ces archives peuvent se présenter sous la forme d'un calendrier, les billets étant classés par date, ou sous la forme d'une liste catégorisée, les billets étant, dans ce cas, classés par thèmes.

3) Puis, une liste de liens vers d'autres sites web et blogs est proposée: le blogroll. Ces liens permettent, alors, de trouver d'autres ressources sur les mêmes thèmes que ceux traités dans le blog. C'est à travers le blogroll que les blogs se lient les uns aux autres, créant un petit réseau au sein du Réseau.

4) Enfin, une possibilité de syndication de contenu, sous la forme d'un lien vers un fichier au format XML reprenant le contenu du site, est offerte sur chaque blog.

\section{3}


De même, les blogs se caractérisent par une grande liberté de ton, une forte personnalisation du site, et un très haut niveau d'interactivité qui privilégie l'instantané et la spontanéité. Bien que proche du concept de wiki, le blog se différencie de ce dernier par le fait que tous les blogeurs ne sont pas égaux sur un blog. En effet, sur un site wiki chaque page peut être modifiée par n'importe quel internaute et ce de manière immédiate ; tandis que sur un blog, l'auteur est le seul à pouvoir modifier le contenu de son site. Les autres blogueurs, en effet, ne peuvent qu'émettre des commentaires sur les billets (les billets ne pouvant être écrits que par les blogeurs ayant le statut de « rédacteur » ou l'auteur du blog). Les commentaires laissés par les blogueurs sont, aussi, soumis au jugement du responsable du blog qui choisit seul de les conserver ou de les supprimer. Ce dernier peut aussi choisir de «promouvoir» d'autres blogueurs au rang de "rédacteurs », ceux-ci bénéficiant, alors, des mêmes droits que lui. Dans ce cas, le blog prend alors une dimension collective. De plus, contrairement au blog, le wiki grossit de façon désordonnée et sa structure est très rarement hiérarchisée ; tandis que le blog laisse à l'auteur un contrôle total de la ligne éditoriale, l'unicité de la source favorisant la cohérence de l'ensemble. (Garreau,2005,11)

Enfin, les blogs font parti de la famille des outils de gestion de contenu appelés CMS (Content Management System). Avant les blogs, les CMS étaient des outils complexes nécessitant d'avoir un minimum de connaissances informatiques. Avoir son propre CMS pouvait, donc, être coûteux tant en temps que financièrement, et bien qu'ils se soient beaucoup simplifiés, les blogs restent les seuls CMS véritablement grand public. En effet, les blogs stockent leur contenu en base de données ce qui permet de séparer le contenu, de l'information liée à la mise en forme. Un intérêt non négligeable à l'heure de la réflexion autour du web sémantique. (Garreau,2005,12)

\section{4}


Toutes ces caractéristiques, expliquent, en partie le succès de ces nouveaux outils de publication très simples et très complets, qui se développent, aujourd'hui, sous de nombreuses formes, touchant à des domaines variés.

\section{Blogs : les raisons du succès}

On ne peut pas, pour autant, nier l'immense succès de cet outil, les raisons de cet engouement étant liées à plusieurs facteurs convergents :

D'abord, la gratuité et la facilité : " le mode de publication est simple et tout se déroule à partir d'une interface semblable à celle de l'envoi d'un courrier électronique ».( Ertzcheid,2005,8)

Puis, l'archivage et l'indexation automatiques, chaque post est indexé à l'aide de balises indiquant l'auteur, la date de publication et le titre. Darlène Fitcher, documentaliste, résume, d'ailleurs, le succès des blogs en ces termes : « la puissance du blog se situe non seulement dans la facilité d'éditer le nouveau contenu, mais également dans sa capacité d'archiver automatiquement de vieux post et de régénérer la teneur de la page principale ».( Fitcher ,2003,19)

A cela s'ajoute aussi le format des blogs. Comme le rappelle Benoit Desavoye

« Le format court des blogs n'est pas nés d'eux ; ce format s'est diffusé lors de l'utilisation de Usernet puis des fora ou du chat. Cependant, le format « post» des blogs a joué un rôle essentiel dans leur succès ».( Desavoye\&Ducamp et al.,2005,15)

En effet, les blogs proposent, un juste milieu entre le format très court des fora et le format plus long, se rapprochant de celui des articles de presse, des sites web. Cependant, le point commun à tous les weblogs, et son plus grand facteur de succès, reste indubitablement la facilité de création et la possibilité d'interaction qu'il offre. (Ertzcheid, 2005.p,8)

\section{5}


Le weblog permet de partager des informations et des fichiers en les mettant à disposition des internautes. Cet outil de publication autorise une dynamicité beaucoup plus importante qu'un site web traditionnel. Il est interactif; l'internaute lecteur peut commenter les écrits du blog via un formulaire en ligne et ce jeu de va-et-vient permet d'établir une communication entre l'auteur et ses lecteurs, créant ainsi une véritable communauté virtuelle.

La réactivité est l'une des principales qualités du blog. Les billets et les commentaires peuvent être postés rapidement depuis n'importe quel ordinateur connecté à Internet et doté d'un navigateur web.(Journal Officiel de l'administration française ,2015)

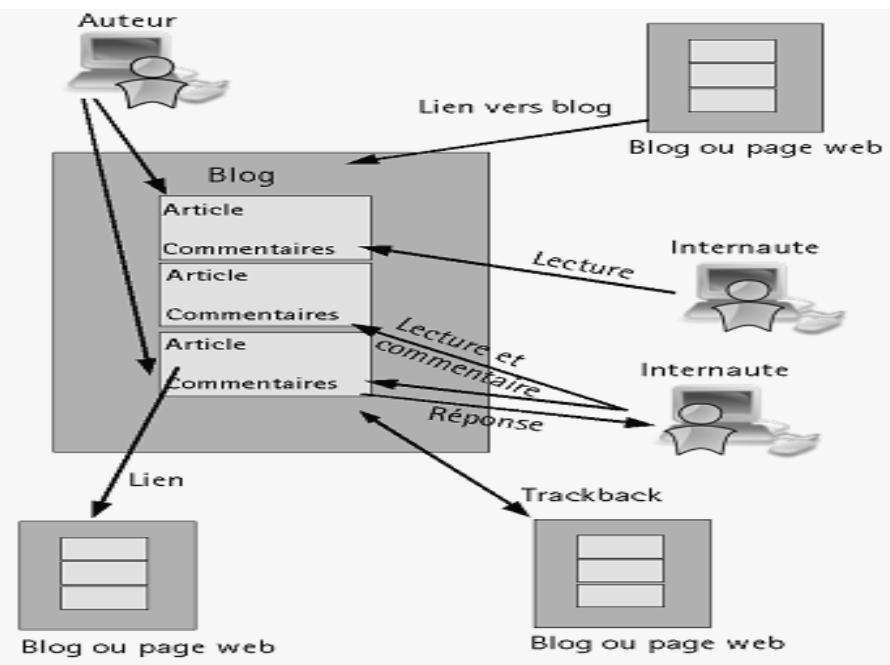

Figure (7) Principes de fonctionnement de la blogosphère.

Francparler (2015) affirme que les enseignants sont également de plus en plus nombreux à tirer parti de l'intérêt suscité par cet outil auprès des jeunes en l'intégrant dans leurs pratiques de classe et en exploitant leur potentiel pédagogique et explique les spécificités du blog par rapport à un site web dans le tableau suivant :

\section{6}

Journal of Arabic Studies in Education \& Psychology(ASEP) 
Tableau (4). Spécificités du blog par rapport à un site web

\begin{tabular}{|c|c|}
\hline Simplicité & $\begin{array}{l}\text { Créer, administrer et mettre à jour un blog est à la portée de } \\
\text { tous. }\end{array}$ \\
\hline Rapidité & $\begin{array}{c}\text { Montre en main, il ne faut pas plus de } 5 \text { minutes pour créer } \\
\text { un blog. La publication de nouveaux articles est également } \\
\text { immédiate. }\end{array}$ \\
\hline Interactivité & $\begin{array}{l}\text { Le blog permet une interactivité entre le créateur du blog et } \\
\text { ses lecteurs. Ceux-ci ont en effet la possibilité de réagir aux } \\
\text { messages de l'auteur, d'apporter leur avis sur le blog en } \\
\text { général ou de lire les réactions des autres visiteurs. Les } \\
\text { interactions peuvent alors prendre l'aspect d'une discussion } \\
\text { entre l'auteur et le lecteur. }\end{array}$ \\
\hline Interconnexion & $\begin{array}{c}\text { Les blogs contiennent de multiples liens vers d'autres } \\
\text { sources d'information sur la Toile : des sites Web, mais } \\
\text { aussi d'autres blogs. Il se crée ainsi une sorte de maillage } \\
\text { entre blogs. }\end{array}$ \\
\hline Gratuité & $\begin{array}{l}\text { De nombreux sites proposent de créer gratuitement un blog. } \\
\text { Cela inclut la création du site, l'édition et l'hébergement du } \\
\text { blog. }\end{array}$ \\
\hline Dynamique & $\begin{array}{c}\text { Une des différences majeures entre un blog et une page web } \\
\text { est la simplicité avec laquelle le blog peut être mis à jour. } \\
\text { Les blogs sont ainsi fréquemment actualisés et leurs archives } \\
\text { restent consultables. }\end{array}$ \\
\hline Accessibilité & $\begin{array}{l}\text { Un blog peut-être ouvert à tous les lecteurs de l'Internet ou } \\
\text { réservé aux membres d'une communauté. }\end{array}$ \\
\hline Auteurs & $\begin{array}{l}\text { On distingue plusieurs niveaux dans la gestion d'un blog: } \\
\text { l'administrateur (qui gère les paramètres de l'outil), l'auteur } \\
\text { (qui publie des articles) et le visiteur (qui peut consulter le } \\
\text { blog et ajouter des commentaires aux différents articles). Un } \\
\text { blog peut avoir plusieurs auteurs. }\end{array}$ \\
\hline
\end{tabular}

\section{Blog dans le cadre de la classe}

Dans le cadre de la classe, l'enseignant peut développer via le blog un espace de consultation et d'accompagnement pour ses étudiants.Les enseignants de langue sont de plus en plus nombreux à utiliser les blogs dans le cadre de leur enseignement et à reconnaître les intérêts pédagogiques que leurs usages, très variés, induisent.

- Pour l'enseignant

- Pour la classe

- Pour l'apprenant

- Blogs et evaluation (Haydée,2015)

\section{7}




\subsection{Pour l'enseignant}

\section{A)Fournir des informations pratiques sur le cours}

Le blog peut servir de support pour fournir des informations pratiques sur les cours telles que le rappel des devoirs, les sujets à venir, le planning des cours, etc.

\section{B)Prolonger le travail réalisé en classe}

Le blog peut également constituer un outil complémentaire au travail réalisé en classe sur lequel les apprenants peuvent se rendre depuis leur ordinateur personnel ou depuis les postes informatiques de l'établissement. Le professeur peut ainsi utiliser le blog comme support de cours dans lequel il publie des résumés de son cours ou propose une trace écrite du contenu le plus important à retenir. L'enseignant peut aussi proposer sur un blog des ressources en ligne commentées, des activités pédagogiques ou des conseils de lecture qui complèteront les sujets abordés en classe, ou encore insister sur une compétence particulière (la lecture, l'écriture...).

\section{C)Proposer des activités pédagogiques}

Le blog peut également être utilisé comme espace d'enseignement sur lequel les étudiants réalisent des tâches ou des scénarios pédagogiques proposés et accompagnés par l'enseignant (un parcours sur Internet, l'étude d'un genre journalistique, des activités de vocabulaire, des jeux pour travailler la grammaire, etc.). L'enseignant peut aussi proposer sur un blog des activités destinées à être réalisées en autonomie par les apprenants.

Le blog peut également être un support motivant pour développer plus particulièrement la compétence d'expression écrite. L'enseignant peut ainsi inviter ses apprenants à publier des commentaires ou des billets sur différentes questions : commentaire d'une citation, réaction à la lecture d'un texte en ligne proposé par le professeur, dictionnaire de classe (le professeur propose chaque

\section{8}

Journal of Arabic Studies in Education \& Psychology(ASEP) 
semaine une expression idiomatique, les étudiants doivent écrire/inventer la définition, trouver des exemples contextualisés ou encore proposer un équivalent en langue maternelle).

\subsection{Pour la classe}

Le blog classe est le résultat d'un travail collaboratif de la classe. Plusieurs utilisations sont possibles :

\section{Raconter la vie de la classe}

Le blog peut servir de cahier de classe dans lequel peuvent être affichés les activités réalisées en classe (production d'activités communes, exemples de travaux d'élèves) éventuellement accompagnées de photos commentées, l'histoire de la classe, le calendrier, les sujets des devoirs à rendre, etc.

\section{Réaliser un projet pédagogique}

Par leurs fonctionnalités, les blogs favorisent le partage des connaissances et la construction coopérative de projets pédagogiques entre étudiants ou entre les membres d'une communauté éducative. En effet, chaque participant peut créer des messages et répondre aux autres, contribuer à l'enrichissement et à la mise à jour des informations dans un même contexte de travail. Le blog permet également à chacun de disposer à tout moment d'une vue globale de l'ensemble des informations. Le blog peut constituer un outil intéressant pour des projets tels que la création d'une newsletter de la classe regroupant les articles écrits par les étudiants et leurs photos, une radio (en utilisant la technologie du Podcast), un journal de classe, un roman photo, Le blog se prête particulièrement bien à des projets d'écriture.

\section{Communiquer}

Les fonctionnalités offertes par les blogs (notamment la fonction "commentaire") favorisent l'interaction et la communication. Communication professeurs/élèves .Le

\section{9}

Journal of Arabic Studies in Education \& Psychology(ASEP) 
blog peut servir d'espace de communication entre professeur et élèves, par exemple pour mettre en évidence des problèmes spécifiques apparus en classe et en discuter avec les élèves, réfléchir sur le déroulement des cours ou encore poursuivre les discussions entamées en classe. L'enseignant peut également proposer des permanences virtuelles pendant lesquelles les élèves peuvent lui poser des questions. Communication élèves/élèves

Le blog peut également être utilisé comme un espace de rencontre où les apprenants de la classe peuvent interagir les uns avec les autres. On peut par exemple imaginer la création de discussions filées, avec des responsabilités (poster sur la liste de discussion, publier des ressources, etc.). L'intérêt est qu'il permet de développer l'esprit de groupe et de libérer la parole des élèves les plus timides.

\section{Communication élèves/extérieur}

Le blog offre de nouvelles possibilités pour la correspondance de classe dans la mesure où il peut constituer un espace virtuel d'échange entre classes. Le blog peut également être utilisé pour la création d'un colloque virtuel avec invités, débats, actes et archives ou encore service d'outil d'interaction avec un invité mystère (professeur, restaurateur, écrivain, artiste...) dont les apprenants doivent deviner l'identité en posant des questions.

\section{3. Pour l'apprenant}

L'enseignant peut encourager chaque apprenant à créer son blog de façon libre ou encadrée. Plusieurs utilisations sont encore une fois envisageables :

\section{Évaluer son travail tout au long de l'année}

Les apprenants peuvent utiliser le blog comme portfolio personnel dans lequel ils pourront publier leurs travaux, enregistrer leur expérience en classe ou leurs réactions face à leur processus d'apprentissage. Le blog constitue dans ce cas un outil d'analyse réflexive et d'auto-évaluation.

\section{0}




\section{Faire ses devoirs}

L'enseignant pourra également inviter les apprenants à se servir de leur blog comme cahier virtuel dans lequel ils peuvent intégrer par exemple des comptes rendus de lecture, donner leur avis sur des thèmes traités en classe en utilisant le vocabulaire et des expressions apprises en cours ou encore documenter une recherche sur Internet (les élèves peuvent y ajouter des liens et des photos, classifier, organiser leur matériel).

\section{Créer un blog en francais sur un sujet de son choix}

L'enseignant peut également encourager l'apprenant à créer son blog personnel dans lequel il pourra s'exprimer dans la langue cible sur des thèmes qui l'intéressent.

\subsection{Blogs et évaluation}

Le blog offre plusieurs possibilités d'évaluation :

- l'auto-évaluation (portfolio)

- la correction par ses pairs

- la correction par l'enseignant (en amont de la publication ou rétrospectivement via la fonction commentaire);

- la correction par d'autres blogueurs

Après avoir présenté les caractéristiques et les avantages de blog, on peut alors, se demander si le blog peut devenir un outil de développement des concepts en recherche en éducation? Nous interroger sur son potentiel en terme de recherche en sciences de l'éducation. Répondre à cette question, nécessite l'appliquer dans la matière " Fondements de recherches" selon les étapes de l'étude expérimentale.

\section{B. La deuxième étape, L'étude expérimentale}

On propose à présent une liste des concepts de recherche en éducation et un test pour mesurer le développement des concepts en recherche en éducation chez les étudiants des études supérieures, diplôme professionnel et un blog composé essentiellement des billets, des blocs ou modules, 
annexes: le dossier de l'étudiant, des éléments graphiques (logo, photos, etc.). Il contient aussi bien du texte, que des images, tous au format numérique. Enfin, il est ouvert sur le reste du Web avec de nombreux liens hypertextes venant étayer ou documenter les propos. L'expérimentation se déroule comme suit :

1) Application du pré-test ;

2) Enseignement de "Fondements de Recherche" selon le blogue proposé ;

3) Application du post-test;

4) Détermination des moyens de traitement statistique ;

5) Interprétation des résultats.

\section{Pour élaborer les outils, on a procédé comme suit:}

1) Définir les concepts de recherche en éducation;

2) Décrire le questionnaire des concepts en recherche en éducation.(Annexe N. 1)

3) Décrire le test des concepts en recherche en éducation.(Annexe N. 2)

\section{A- Questionnaire des concepts de recherche en éducation.}

Pour élaborer ce questionnaire, on a procédé comme suit:

1) Définir ses objectifs ;

2) Déterminer ses sources ;

3) Décrire ce questionnaire.

A.1.La validation de questionnaire des concepts de recherche en éducation

\section{A. Objectif du questionnaire}

Il vise à déterminer les concepts de recherche en éducation que les étudiants des études supérieures doivent posséder.

\section{B. Sources du questionnaire}

On s'inspire ces concepts dans plusieurs sources:

\section{2}

Journal of Arabic Studies in Education \& Psychology(ASEP) 
- Les références étrangères concernant de recherche en sciences de l' éducation ;

- Les études antérieures, les sites d'Internet et les recherches effectuées dans ce domaine qui sont considérées comme une ressource essentielle.

\section{C- Décrire le questionnaire.}

La chercheuse a élaboré la forme initiale de questionnaire des concepts -clés de recherche en sciences de l'éducation. Ce questionnaire comprend les 8 volets suivants :

1) La notion de recherche;

2) Les objectifs et les questions de recherche;

3) La revue critique de la littérature ;

4) L'opérationnalisation de concepts;

5) Le recueil des données;

6) L'analyse de résultats;

7) La conclusion;

8) Le rapport de recherche.

\section{Présentation du questionnaire au jury.}

Après avoir élaboré le questionnaire, la chercheuse l'a présenté à un jury se composant de :

- certains spécialistes en linguistique moderne ;

- certains spécialistes en didactique du F.L.E ;

\section{Les membres du jury sont invités à :}

- supprimer les items non nécessaires ;

- ajouter les items non inclus ;

- modifier les items, si c'est nécessaire.

D'après les propositions présentées par les membres du jury, on a précisé les concepts les plus importants dans le domaine de recherche en sciences de l'éducation.Le questionnaire des concepts de recherche en éducation

\section{3}

Journal of Arabic Studies in Education \& Psychology(ASEP) 
comprend dans sa forme définitive 8 volets essentiels et ses sous-habiletés dont les sujets de l'échantillon ont besoin selon les propositions présentées et les écrits et les études dans le domaine de recherche en sciences sociales en générale., (Annexe no. 1 )

\section{B- Test des concepts de recherche en éducation}

Pour élaborer ces tests, nous avons procédé comme suit:

- Définir les objectifs de test;

- Déterminer les sources de test ;

- Décrire le test.

\section{A-l'objectif de test}

Il vise à mesurer le développement des concepts en recherche en éducation chez les étudiants des études supérieures de section du français.

Le test a pour but de mesurer la maîtrise des étudiants de:

1) La notion de recherche;

2) Les objectifs et les questions de recherche;

3) La revue critique de la littérature ;

4) L'opérationnalisation de concepts;

5) Le recueil des données;

6) L'analyse de résultats;

7) La conclusion;

8) Le rapport de recherche. . (Annexe no.2 ).

\section{B-Déterminer les sources de test ;}

On s'inspire les questions de test dans plusieurs sources:

- Les références étrangères concernant de recherche en sciences de l' éducation ;

- Les études antérieures, les sites d'Internet et les recherches effectuées dans ce domaine qui sont considérées comme une ressource essentielle.

\section{4}

Journal of Arabic Studies in Education \& Psychology(ASEP) 


\section{C- Décrire le test}

\section{La validation du test.}

Le test a eu pour objectif de contrôler le niveau des sujets de l'échantillon avant et après l'expérimentation. Ce test comprend 8 volets gradués selon les étapes de recherche en éducation On a opéré une étude-pilote pour calculer la durée, la fidélité, la validité et les critères de notation pour le test comme suit :-

- Calcul de la durée du test. On a appliqué le test sur les sujets de l'échantillon et on a noté le temps pris par chaque apprenant puis an a calculé la moyenne de durée prise par les apprenants de l'échantillon selon l'équation ci-dessous :

La durée $=$

Somme (ou bien Total (de temps pris par les apprenants de l'échantillon

No. des apprenants de l'échantillon

On a trouvé que la durée du test est $=330$ minutes .

- Calcul de la fidélité du test. On a appliqué et réappliqué le test dans une intervalle de (15) jours et on a calculé la fidélité selon les équations ci-dessous :- (Fouad AlBahey,1979).

$$
\mathrm{R}=\frac{N \sum X Y-\sum X \sum Y}{\sqrt{\left[N \sum X^{2}-\left(\sum X\right)^{2}\right] \mid N \sum Y^{2}-\left(\sum Y\right)^{2}}}
$$

$\mathrm{R}=$ Le coefficient de corrélation.

$\mathrm{N}=$ Le nombre des étudiants.

$\Sigma=$ La somme.

$\mathrm{X}=$ Les notes des étudiants à la première application.

$\mathrm{Y}=$ Les notes des étudiants à la deuxième application.

$\mathrm{X} 2=$ Les carrés des notes des étudiants à la première

\section{5}

Journal of Arabic Studies in Education \& Psychology(ASEP) 
application.

Y2= Les carrés des notes des étudiants à la deuxième application.

Indice de fidélité $(\mathrm{F})=.\frac{2 \mathrm{R}}{1+\mathrm{R}}=0.821$

Indice de fidélité ( F. ) de la partie des compétences argumentative $=0.821$

- On a calculé aussi la stabilité du test selon (AlphaCronbach).

Tableau (5).La stabilité du test des concepts de recherche en éducation

\begin{tabular}{|c|c|c|c|c|c|c|c|c|}
\hline Item & 1 & 2 & 3 & 4 & 5 & 6 & 7 & 8 \\
\hline $\begin{array}{c}\text { Alpha- } \\
\text { Cronbach }\end{array}$ & 0.65 & $\begin{array}{c}0.71 \\
* *\end{array}$ & $\begin{array}{c}0.58 \\
* *\end{array}$ & $\begin{array}{c}0.76 \\
* *\end{array}$ & $* * 0.72$ & 0.75 & 0.73 & 0.80 \\
\hline
\end{tabular}

On a trouvé que le test est stable puisque l' indice de stabilité selon L'Alpha-Cronbach est (0.80).

\section{Calcul de la validité du test}

Pour calculer la validité du test, on a eu recours à deux moyens :

1) On a présenté le test aux membres du jury. Ils ont décidé que le test est valide à mesurer les compétences évaluées.

2) On a calculé la validité de constance interne du test à travers la corrélation de Bearson (la corrélation entre la note chaque item et la note totale du teste comme suit:-

Tableau (6).La validité interne du test des concepts de recherche en éducation

\begin{tabular}{|c|c|c|c|c|c|c|c|c|}
\hline Item & 1 & 2 & 3 & 4 & 5 & 6 & 7 & 8 \\
\hline $\begin{array}{c}\text { Corrélation } \\
\text { de Bearson }\end{array}$ & 0.78 & $\begin{array}{c}0.76 \\
* *\end{array}$ & $\begin{array}{c}0.71 \\
* *\end{array}$ & $\begin{array}{c}0.66 \\
* *\end{array}$ & $0.669^{* *}$ & 0.81 & 0.76 & 0.72 \\
\hline
\end{tabular}

**Sig au niveau 0.01 
On a trouvé que le test est valide puisque les indices de validité interne du test est signifiant au niveau 0.01

\section{E. Critères de notation pour le test.}

On a consacré (300) notes pour le test en entier. On a corrigé les feuilles des apprenants selon une rubrique de critères de notation conçue à ce propos (Cf. annexe No. (2).

\section{Commentaire général:}

A partir des indices qu' on a retrouvé ,on peut constater que le test concernant les concepts de recherche est valable. Donc il est applicable.

\section{Programme proposé}

\section{1-Objectifs spécifiques du bloque proposé}

Il est prévu que l'étudiant, après l'enseignement à travers le blogue proposé, perfectionnera les concepts concernant :

1) La notion de recherche et de la science;

2) L'objectifs et les questions de recherche;

3) La revue critique de la littérature ;

4) L'opérationnalisation de concepts;

5) Le recueil des données;

6) L'analyse de résultats;

7) La conclusion;

8) Le rapport de recherche.

\section{2-Le contenu et l'organisation du programme:}

Le blogue proposé comprend les éléments suivants:

- La définition des objectifs concernés ;

- l'explication de la tâche;

- le contenu à enseigner ;

- Les approches utilisées ;

- le dossier de l'étudiant: consignes, critères, et document; activités ;\& exercices.

\section{7}

Journal of Arabic Studies in Education \& Psychology(ASEP) 
- des liens hypertextes venant étayer ou documenter les propos (Annexe no.3)

Le professeur serait un coordinateur, un animateur, un guide et un "contrôleur" qui pourrait aider les apprenants à trouver la bonne voie. Ainsi l'apprenant prend l'habitude d'exercer sa créativité, son intuition et sa spontanéité .

\section{Choix de l'échantillon :}

Nous avons choisi au hasard un échantillon de 30 chercheurs parmi les étudiants des études supérieures, diplôme professionnel, de section de français à la faculté de pédagogie de Menoufya.

\section{La durée de l'enseignement des unités :}

L'enseignement de ces unités a duré le 1er trimestre de l'année (2015/2016). Pendant cette durée, nous avons enseigné les unités proposées de la matière " Fondement de la recherche" selon le blogue proposé . Durant l'expérience, les étudiants étaient actifs. Il y avait une interaction fructueuse soit entre les étudiants les uns les autres ou entre les étudiants et leur enseignante.

\section{L'application du post-test.}

A la fin de la période consacrée à l'enseignement du programme, on a réappliqué le (pré - post) test des concepts aux membres de l'échantillon afin de déterminer le degré de développement des étudiants des études supérieures de section de français concernant les concepts de recherche . Ainsi on a passé à l'analyse statistique des résultats.

\section{Analyse des résultats:}

La chercheuse a utilisé la version SPSS (version $1^{\wedge}$ ) en traitant les résultats pour tester la validité des hypothèses de la recherche

\section{Première hypothèse:}

Il y a une différence statistiquement significative entre les moyennes des notes des étudiants du groupe expérimental au pré-test et au post-test des concepts de recherche en

\section{8}

Journal of Arabic Studies in Education \& Psychology(ASEP) 
sciences de l'éducation au niveau 0.01 en faveur du post test;

Pour vérifier cette hypothèse,on a calculé la valeur de $(\mathrm{T})$ $\& \eta$ pour examiner l'existence d'une différence entre les moyennes des notes des étudiants du groupe expérimental au pré-test et au post-test des concepts.

Tableau (7). Résultats descriptif relatifs au (pré -post )test des concepts pour le groupe expérimental

\begin{tabular}{|c|c|c|c|c|c|}
\hline Concepts & Application & $\mathrm{N}$ & M. & E.T & $\begin{array}{l}\text { Points accordés a } \\
\text { chaque concept }\end{array}$ \\
\hline \multirow{2}{*}{ Notion de recherche } & Pré-test & 30 & 17.97 & 4.57 & \multirow{2}{*}{ 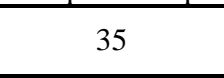 } \\
\hline & Post-test & 30 & 32.30 & 3.34 & \\
\hline \multirow{2}{*}{$\begin{array}{l}\text { Objectifs et Questions de } \\
\text { recherche }\end{array}$} & Pré-test & 30 & 18.40 & 4.01 & \multirow{2}{*}{35} \\
\hline & Post-test & 30 & 32.03 & 3.05 & \\
\hline \multirow{2}{*}{$\begin{array}{c}\text { Revue critique de la littérature } \\
\text { \& Cadre conceptuel }\end{array}$} & Pré-test & 30 & 11.10 & 4.67 & \multirow{2}{*}{25} \\
\hline & Post-test & 30 & 22.47 & 3.66 & \\
\hline \multirow{2}{*}{$\begin{array}{c}\text { Opérationnalisation des } \\
\text { concepts }\end{array}$} & Pré-test & 30 & 29.93 & 6.52 & \multirow{2}{*}{60} \\
\hline & Post-test & 30 & 54.37 & 6.54 & \\
\hline \multirow{2}{*}{ Recueil des données } & Pré-test & 30 & 15.53 & 5.66 & \multirow{2}{*}{35} \\
\hline & Post-test & 30 & 31.53 & 2.69 & \\
\hline \multirow{2}{*}{ Analyse des résultats } & Pré-test & 30 & 16.90 & 6.18 & \multirow{2}{*}{30} \\
\hline & Post-test & 30 & 27.90 & 2.40 & \\
\hline \multirow{2}{*}{ Conclusion } & Pré-test & 30 & 6.30 & 2.00 & \multirow{2}{*}{15} \\
\hline & Post-test & 30 & 13.13 & 1.36 & \\
\hline \multirow{2}{*}{ Rapport de recherche } & Pré-test & 30 & 31.30 & 7.71 & \multirow{2}{*}{65} \\
\hline & Post-test & 30 & 60.83 & 5.06 & \\
\hline \multirow{2}{*}{ Total } & Pré-test & 30 & 147.43 & 20.73 & \multirow{2}{*}{300} \\
\hline & Post-test & 30 & 274.57 & 10.52 & \\
\hline
\end{tabular}

Tableau (8). Résultats du groupe expérimental au pré / post test

\begin{tabular}{|c|c|c|c|c|c|c|c|}
\hline \multirow{2}{*}{ Concepts } & \multicolumn{2}{|c|}{$\begin{array}{c}\text { Différences des } \\
\text { moyennes }\end{array}$} & \multirow{2}{*}{$\begin{array}{c}\mathrm{T} \\
\text { Calculé }\end{array}$} & \multirow{2}{*}{ d. } & \multirow[t]{2}{*}{${ }^{2} \eta$} & \multirow{2}{*}{$\begin{array}{l}\text { Effec } \\
\text { t size } \\
\text { (d) }\end{array}$} & \multirow{2}{*}{ Sig } \\
\hline & $\begin{array}{c}\mathrm{La} \\
\text { moyenne }\end{array}$ & $\begin{array}{l}\text { L'écart type } \\
\text { (E.T) }\end{array}$ & & & & & \\
\hline Notion de recherche & 14.33 & 5.50 & 14.262 & 29 & 0.88 & 5.30 & $\begin{array}{l}\text { Sig. \& Très } \\
\text { important }\end{array}$ \\
\hline $\begin{array}{l}\text { Objectifs et Questions } \\
\text { de recherche }\end{array}$ & 13.63 & 4.37 & 17.101 & 29 & 0.91 & 6.35 & $\begin{array}{c}\text { Sig. \& Très } \\
\text { important }\end{array}$ \\
\hline $\begin{array}{l}\text { Revue critique de la } \\
\text { littérature \& Cadre } \\
\text { conceptuel }\end{array}$ & 11.37 & 6.54 & 9.525 & 29 & 0.76 & 3.54 & $\begin{array}{l}\text { Sig. \& Très } \\
\text { important }\end{array}$ \\
\hline $\begin{array}{l}\text { Opérationnalisation des } \\
\text { concepts }\end{array}$ & 24.43 & 5.73 & 23.341 & 29 & 0.95 & 8.67 & $\begin{array}{l}\text { Sig. \& Très } \\
\text { important }\end{array}$ \\
\hline Recueil des données & 16.00 & 5.67 & 15.459 & 29 & 0.89 & 5.74 & $\begin{array}{c}\text { Sig. \& Très } \\
\text { important }\end{array}$ \\
\hline Analyse des résultats & 11.00 & 6.99 & 8.616 & 29 & 0.72 & 3.20 & $\begin{array}{l}\text { Sig. \& Très } \\
\text { important }\end{array}$ \\
\hline Conclusion & 6.83 & 2.12 & 17.666 & 29 & 0.91 & 6.56 & $\begin{array}{l}\text { Sig. \& Très } \\
\text { important }\end{array}$ \\
\hline Rapport de recherche & 29.53 & 10.66 & 15.17 & 29 & 0.89 & 5.63 & $\begin{array}{c}\text { Sig. \& Très } \\
\text { important }\end{array}$ \\
\hline Total & 127.13 & 27.31 & 25.496 & 29 & 0.96 & 9.47 & $\begin{array}{l}\text { Sig. \& Très } \\
\text { important }\end{array}$ \\
\hline
\end{tabular}


N: Nombre des étudiants. M : Moyenne arithmétique E.T : Ecart type D.L. : Degré de liberté T: Valeur de T. ( $\left.\eta^{\prime}\right)$ : l'ETA - carré. Sig: Seuil de signification

\section{Commentaire :}

on a observé que la valeur de (t ) calculée est significative au seuil de (0.01). Ce qui a confirmé que la première hypothèse est vérifiée. on a observé que la valeur de $\left({ }^{2} \eta\right)$ est (0.96), une grande valeur .Cela indique que le (96\%) des différences entre les moyennes des notes du groupe expérimental au pré-post test renvoient au blogue désigné.

On peut résumer ces résultats dans les figures suivantes qui nous montre la différence entre les moyennes des notes des étudiants du groupe expérimental au pré -post -test en faveur du post-test.

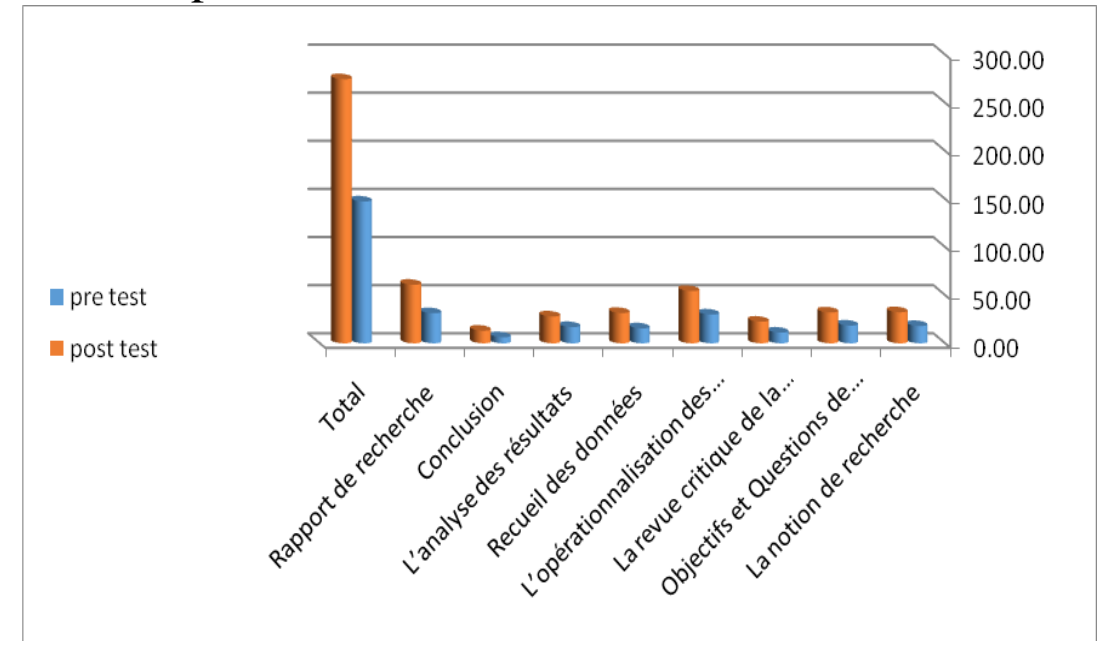

Figure 8.Bar Chartes de la moyenne arithmétique de pré- post test des concepts de recherche.

Tableau (9). Les boîtes à moustache

\begin{tabular}{|c|c|c|}
\hline Mesures Statistiques & pré & post \\
\hline Minimum & 119 & 234 \\
\hline Maximum & 188 & 275 \\
\hline quartile inférieur & 132.75 & 253.75 \\
\hline Médiane & 140 & 266 \\
\hline quartile supérieur & 167.25 & 272 \\
\hline
\end{tabular}




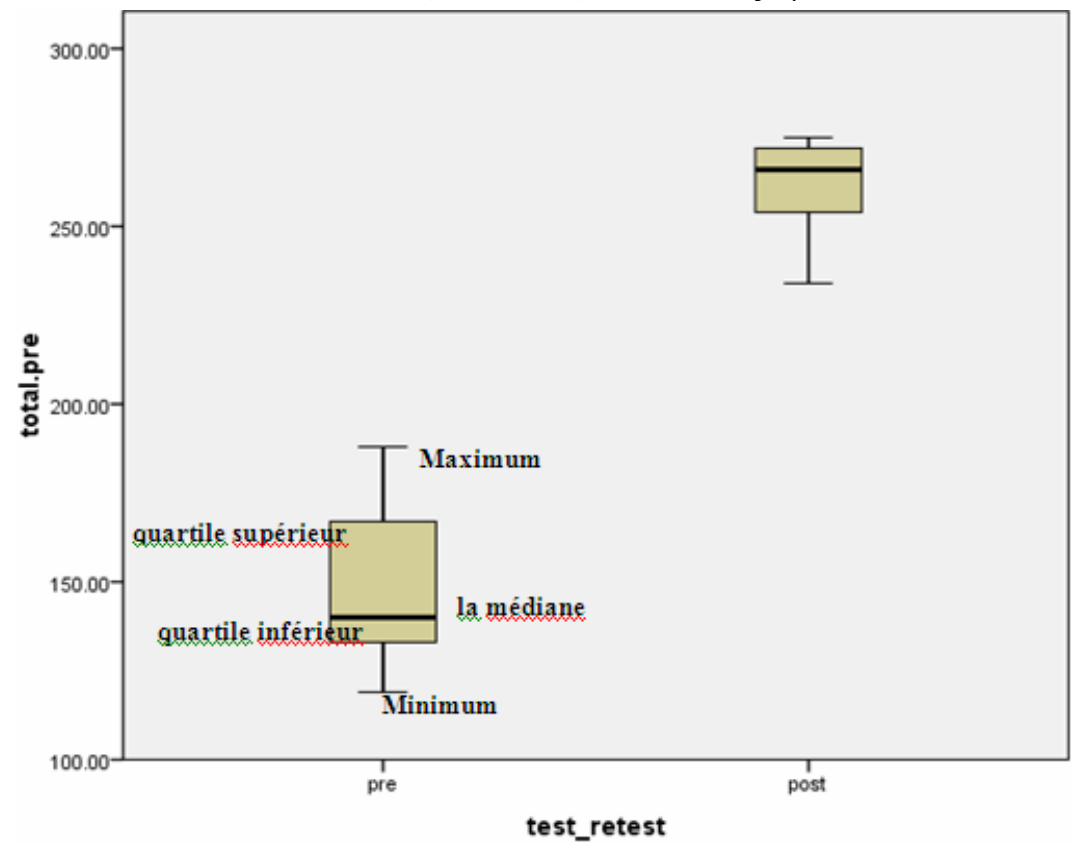

Figure(9). Les boîtes à moustache

Pour vérifier la dernière hypothèse, on a calculé le pourcentage du gain modifié de Black. On a obtenu les résultats qui figurent dans le tableau suivant:

Tableau No.10. Le gain modifié de Black relatif aux concepts de recherche en éducation

\begin{tabular}{|c|c|c|c|c|}
\hline & $\begin{array}{c}\text { Différence } \\
\text { entre les 2 } \\
\text { moyennes }\end{array}$ & $\begin{array}{c}\text { Note } \\
\text { totale. }\end{array}$ & $\begin{array}{c}\text { gain } \\
\text { modifié } \\
\text { de Black }\end{array}$ & Sig. \\
\hline Notion de recherche & 14.33 & 35 & 1.25 & Sig. \& important \\
\hline $\begin{array}{c}\text { Objectifs et les } \\
\text { questions de } \\
\text { recherche }\end{array}$ & 13.63 & 35 & 1.21 & Sig. \& important \\
\hline $\begin{array}{c}\text { Revue critique de la } \\
\text { cittérature \& Cadre } \\
\text { conceptuel }\end{array}$ & 11.37 & 25 & 1.27 & Sig. \& important \\
\hline $\begin{array}{c}\text { Opérationnalisation } \\
\text { des concepts }\end{array}$ & 24.43 & 60 & 1.22 & Sig. \& important \\
\hline Recueil des données & 16.00 & 35 & 1.28 & Sig. \& important \\
\hline $\begin{array}{c}\text { Analyse des } \\
\text { résultats }\end{array}$ & 11.00 & 30 & 1.21 & Sig. \& \\
\hline important \\
\hline $\begin{array}{c}\text { Ranclusion } \\
\text { rechert de }\end{array}$ & 6.83 & 15 & 1.24 & Sig. \\
\hline Total & 127.13 & 300 & 1.26 & Sig. \& important \\
\hline
\end{tabular}




\section{Commentaire :}

Ce tableau montre que le pourcentage du gain modifié de Black est (1.26), cette valeur est plus élevée que la valeur fixée (1.20).Ceci prouve l'efficacité du Weblog sur le développement des concepts de la recherche en sciences de l'éducation chez les étudiants des études supérieures,diplôme professionnel, à la faculté de pédagogie.

\section{Commentaire général des tableaux:}

- En examinant les tableaux (7),(8),(9)\&(8), on remarque que il y a une différence statiquement significative entre les moyennes des notes des étudiants du groupe expérimental au pré -test et au post test au niveau 0.01 en faveur du post-test. Et le (96\%) des différences entre les moyennes des notes du groupe expérimental au pré-post test renvoient au blogue désigné.

- Le blogue proposé a une grande efficacité sur le développement des concepts de recherche en éducation .

\section{Analyse Qualificative\&Interprétation des résultats}

On peut dire que les résultats et la justification des hypothèses de la recherche reviennent aux éléments suivants:

1) A travers du suivi des étudiants en diplôme professionnel, le blogue a montré sa efficacité: le blogue a permis une réflexion plus construite et individuelle des travaux menés pendant l'étude. Il nous semble intéressant de citer les huit dimensions explorées par cet outil:

- le gout d'apprendre;

- la perception de soi en tant qu'apprenant efficace;

- l'acceptation de la complexité;

- -la créativité;

- -la conception de l'éducation comme permanente et bénéfique;

\section{2}


- L'initiative dans l'apprentissage;

- l'autonomie;

- l'accaptation de sa responsabilité dans l'apprentissage.

Ces résultats sont en accord avec Lebrun $(2002,186)$ qui affirme que « les bénéfices les plus flagrants de l'intégration des technologies de l'information et de la communication dans l'enseignement sont à rechercher parmi les méthodes ouvertes et actives [dont font partie le blogue], car elles contribuent à la préparation des personnalités "fortes" que la société revendique »;

2) Le blogue proposé a été créé pour aider tous ceux qui, dans le cadre de leurs études, de leurs responsabilités professionnelles, souhaitent se former à la recherche en éducation ou, plus précisément, entreprendre avec succès des travaux, des analyses ou des recherches dont l'objectif est de comprendre plus profondément et d'interpréter plus justement les phénomènes de la vie éducative auxquels ils sont confrontés;

3) Le blogue proposé a été conçu comme un support de formation méthodologique au sens large, c'est-à-dire comme une formation à la conception et à la mise en œuvre d'un dispositif d'élucidation du réel. Cela signifie que on aborde dans un ordre logique des thèmes tels que la formulation d'un projet de recherche, la construction d'un plan de recherche ou les critères de choix des techniques de recueil, de traitement et d'analyse des données;

4) Il se présente donc comme un manuel dont les différentes parties peuvent être expérimentées, soit par des apprentis chercheurs isolés, soit en groupe ou en salle de cours.

5) Le blogue ou bien le contenu de blogue proposé ne se présente pas comme un simple collection de recettes mais bien comme un canevas général et très ouvert dans le cadre duquel les démarches concrètes les plus variées peuvent être mises en œuvre.

\section{3}


6) Il contient effectivement de nombreuses suggestions pratiques et des exercices d'application. C'est une voie méthodologique précise et irrévocable.

7) Le blogue proposé a été créé pour aider le chercheur à concevoir par lui-même une démarche de travail . Il n'est donc pas un " mode d'emploi" qui impliquerait une application mécanique de ses différentes étapes.

8) Il propose des repères aussi polyvalents que possible pour que chacun puisse élaborer lucidement ses propres dispositifs méthodologiques, en fonction de ses propres objectifs.

9) Le contenu et les activités de blogue proposé invitent constamment au recul critique, de sorte que le chercheur soit régulièrement amené à réfléchir avec lucidité au sens de son travail au fur et à mesure de sa progression.

\section{Recommandations de la recherche}

À la lumière des résultats obtenus par cette recherche, on peut proposer les recommandations suivantes:

1) Utiliser le blog comme support de cours dans lequel l'enseignant peut publier des résumés de son cours ou proposer une trace écrite du contenu le plus important à retenir, fournir des informations pratiques sur les cours telles que le rappel des devoirs, les sujets à venir, le planning des cours, etc.

2) Proposer sur un blog des ressources en ligne commentées, des activités pédagogiques ou des conseils de lecture qui complèteront les sujets abordés en classe, ou encore insister sur une compétence particulière (la lecture, l'écriture...).

3) Proposer des activités pédagogiques sur un blog comme (un parcours sur Internet, l'étude d'un genre journalistique, des activités de vocabulaire, des jeux pour travailler la grammaire, etc.).

\section{4}

Journal of Arabic Studies in Education \& Psychology(ASEP) 
4) Susciter chez les apprenants l'envie d'utiliser les blogues, encourager les apprenants à poster sur le blog leurs devoirs au lieu de les remettre uniquement à l'enseignant, participer à l'alimentation du blog et faire de cette activité un devoir de classe,etc.

5) Encourager l'apprenant à créer son blog personnel dans lequel il pourra s'exprimer dans la langue cible sur des thèmes qui l'intéressent. L'apprenant pourra par exemple rédiger un compte rendu hebdomadaire sur les activités réalisées dans le cadre de la classe, publier des commentaires sur le contact personnel qu'il a eu avec la langue cible hors du contexte classe (les chansons, la télévision, le cinéma, les voyages), décrire des moments de sa vie de tous les jours, parler de sa région, de son pays, de ses loisirs, commenter l'actualité, des articles/livres, un voyage, recommander des films, des chansons ou des jeux ou encore inviter d'autres personnes à réagir sur des thèmes qui l'intéressent.

6) Utiliser le blog comme portfolio personnel dans lequel les apprenants pourront publier leurs travaux, enregistrer leur expérience en classe ou leurs réactions face à leur processus d'apprentissage.

\section{Suggestions de la recherche}

À la lueur des résultats de la recherche, on peut suggérer les recherches suivantes:

1) Étudier l'efficacité des blogs en vue de développer les compétences d'analyse réflexive et d'auto-évaluation;

2) Étudier l'efficacité des blogs en vue de libérer la parole des élèves les plus timides ou bien en vue de développer les compétences de communication;

3) Étudier l'efficacité des blogs en vue de développer la compétence de la communication écrite, l'écriture collective \& académique, créative, littéraire etc.

4) Utiliser des blogs en vue de construire un projet pédagogique collaboratif entre étudiants ou entre les membres d'une communauté éducative;

\section{5}

Journal of Arabic Studies in Education \& Psychology(ASEP) 
5) Utiliser le « visiochat » avec le blogue pour développer la créativité et les compétences de communication chez les apprenants;

6) Étudier l'efficacité des blogs en vue de développer la mise en œuvre de la gestion des connaissances (Enseigner/Diffuser, Piloter/Analyser, Appliquer/Réaliser une activité).

\section{Bibliographie.}

- American Education Research Association [Association américaine pour la recherche sur l'éducation] (AERA), (2006).La notion de recherche. Washington.

- Angeline, A-L. (2007). Études et conseils : démarches et outils (enligne http://www.esen.education.fr/ conseils/commande/ operations/formuler-une-problematique

- Asselin, M. (2006). «Les blogues: de puissants outils pour faire apprendre ». SavoirsCDI. Consulté en février 2008: http://savoirscdi.cndp.fr/pedago/ Blogs/MarioAsselin.pdf

- Bachelard, G. (1965).La formation de l'esprit scientifique. Paris, Librairie philosophique J. Vrin.

- Bachtold M. (2012), «Les fondements constructivistes de l'enseignement des sciences basé sur l'investigation ", Tréma, $n^{\circ} 38, p .7-39$

- Batier, C. (octobre 2005). L'équipement informatique et son usage chez les étudiants de première année sciences de l'Université Lyon 1. Récupéré le 8 juillet 2007 du site (NouvellesTechnologiesÉducatives-Lyon1:http://nte-serveur.univlyonl.fr/coursinfo/ resultats2005. Html).

- Bernatchez, J. (2006). "Le blogue comme instrument d'apprentissage: bilan d'une expérience réalisée à l'Ecole nationale d'administration publique ». ENAP, Université du Québec. Consulté en mai 2008: http://www.uquebec.ca/capres/fchiers/art_ENAP-juin06.shtml

- Berthelot, J-M. (1990)L'intelligence du social, Paris, PUF.

- Bourdieu, P.Chamboredon, J.-C. et Passeron J.-C. (1968).Le métier de sociologue, Paris, Mouton, Bordas.

- Brent, D. (2005). " Trois attitudes dans la recherche en éducation: autour de "l'explicite", de "l'implicite" et de la "complicité" ». Revue des sciences de l'éducation, vol. 31, n², pp. 397-416.

\section{6}




\section{Number 70, Part 2, February, 2016}

- Briggs, G., Shamma, D. A., Cañas, A. J., Carff, R., Scargle, J., \& Novak, J. D. (2004). Concept maps applied to Mars exploration public outreach. In A. J. Cañas, J. D. Novak \& F. González (Eds.), Concept maps: Theory, methodology, technology. Proceedings of the first international conference on concept mapping (Vol. I, pp. 109-116). Pamplona, Spain: Universidad Pública de Navarra.

- Campbell, A. P. (2003). "Weblogs for use with ESL classes". The Internet TESL Journal.Vol.IX,nº2.Consulté en octobre 2007: http://iteslj.org/Techniques/Campbell-Weblogs.html

- Class, B\& Schneider, D (.2015). Manuel de recherche en technologie éducative. Université de Genève, Suisse. publie EduTech Wiki (en-ligne https://edutechwiki.unige.ch/ fr/Manuel_de_recherche_en_technologie_\%C3\%A9ducative

- Cooper, H. (2010). Research synthesis and meta-analysis: A stepby-step approach (4e éd.).Thousand Oaks, CA: Sage.

- Creswell, John W. (2014).Research Design Qualitative, Quantitative, and Mixed Methods Approaches. - SAGE Publications, London.New Delhi.Fourth Edition (en-ligne: https://www.researchgate.net/file.PostFileLoader.html?id...assetKe y...)

- Demange-Ducrot, C. (2006). Les blogs à l'école: Intérêts et enjeux pour le professeur-documentaliste. Mémoire professionnel en CAPES de documentation. Académie de Strasbourg, IUFM d'Alsace. Consulté en novembre 2007: http://savoirscdi.cndp.fr/pedago/Blogs/C.\%20Demange-Ducrot.pdf

- De Ketele, J.-M., Maroy, C. (2006). Quels critères de qualité pour les recherches en éducation? In L., Paquay, M., Crahay \& J.-M., De Ketele, L'analyse qualitative en éducation. Des pratiques $d e$ recherche aux critères de qualité. Bruxelles : De Boeck Universités, coll. Pédagogies en développement, pp. 219-249

- Demers, P. (1994). La formation des chercheurs en éthique. Revue de presse, 12 Avril, 1994, p 83).

- Desavoye, B; Ducamp, C. (2005).Les blogs: nouveau média pour tous. Paris: M2 Editions.

- Deitering, AM., Huston, S. (2004). "Weblogs and the "Middle Space" for Learning". Academic Exchange Quarterly. Winter, Volume 8, Issue 4. Consulté en septembre 2006: http://www.rapidintellect.com/AEQweb/5mar2879z4.htm

- Downes, S. (2005). "E-Learning 2.0". ELearn Magazine (CPublications in Trade Journals (invited article)) Association for Computing Machinery Oc. Consulté en novembre 2007: http://www.downes.ca/post/31741 


\section{Number 70, Part 2, February, 2016}

- Dumez,H.(2010) "Éléments pour une épistémologie de la recherche qualitative en gestion" Le Libellio d'Aegis, vol. 6, $\mathrm{n}^{\circ} 4$, pp. 3-15.

- Dumez, H. (2011). Faire une revue de literature: pourquoi et comment? Le Libellio d' AEGIS. CNRS / École Polytechnique. Vol. 7, n 2 - Eté 2011pp. 15-27

- Durkheim, E. (1991).Les règles de la méthode sociologique, précédé de J.-M. Berthelot, Les règles de méthode sociologique ou instauration du raisonnement expérimental en sociologie, Paris, Flammarion, 1988.

- Ertzcheid, O. (2005). Weblogs: UN nouveau paradigme pour les systèmes d'information et la diffusion de connaissances? : Applications et cas d'usage en contexte de veille et d'intelligence économique. Colloque ISKO France.

- Ertzcheid, O. (2011). Créer, trouver et exploiter lesblogs.Association des professionnels de l'information et de la documentation (ADBS), editions, 64. 2008. Submitted on21Apr2011(en-ligne https://archivesic.ccsd.cnrs.fr/sic $00586027 v 2$ )

- Fievet, C.; Turrettini, E. (2004). Blog story. Paris: Eyrolles.

- Fitcher, D. (2003). Why and How to Use Blogs to Promote Your Library's Services? [En ligne].Décembre 2003. infotoday .Vol. 17 No. 6 - Nov/Dec 2003(http://www.infotoday .com/mls/nov03/fichter.shtml)

- Francparler.(2015). Blogs: quelles applications pédagogiques? Site des professeurs de français (en ligne: http://www.francparleroif.org/ applications-pedagogiques/)

- Garreau, A. (2005). Les blogs entre outil de publication et espace de communication.un nouvel outil pour les professionnels de la documentation: Thèse / Mémoire. Université catholique de l'ouest - ANGERS Institut d'Arts, Lettres et Histoire (https://memsic.ccsd.cnrs.fr/mem_00000273)

- Hart, C. (2009). Doing a literature review: Releasing the social science research imagination, LA/London, Sage.

- Haydée, M. (2015).Qu'est-ce qu'un blog? et comment s'en servir pédagogiquement? Dossier Franc-parler Réalisé à partir de l'article de Francparler (en-ligne: http://www.francparler.org/ parcours/ blogs.htm Page 1).

- Johnson, A. (2004). "Creating a Writing Course Utilizing Class and Student Blogs”. The Internet TESL Journal, Vol. X, $n^{\circ} 8$. Consulté en septembre 2006: http://iteslj.org/Techniques/Johnson-Blogs/ 


\section{Number 70 , Part 2 , February, 2016}

- Journal Officiel de l'administration française (Le 20 mai, 2005).Le Blog.france2.fr (izibook.eyrolles.com/extract/show/4910).

- Journot, E., Lelièvre, C. et Diolosa, V. (13 janvier, 2006,). Synthèse. Récupéré le 8 juillet 2007 du site Équipement informatique des étudiants en Europe : (en ligne:http://infoetudianteuro.canalblog.com/)

- Karsenti, T. \&Savoie-Zajc, L. (2000).Introduction à la recherche en éducation. Sherbrooke, QC: Éditions du CRP.

- Lamoureux, A. (2006). Recherche et méthodologie en science humaines. 2e édition. Groupe Beauchemin, Editions Etudes Viviane's .

- Lara, T. (2006). Usos de los blogs en una pedagogía constructivista. Consulté en décembre 2007: http://unileon.pbwiki.com/fledublogs.pdf

- Le site Pointblog.com16, (2011). Définition du blog. Ertzscheid,2011, Submitted on 14Apr2011 (v1), last revised 21Jan2011(v2) ( en ligne: http://www.lemondedublog.com/abc/ ) http://www.pointblog.com/

- Lebrun, M. (2002). Des technologies pour enseigner et apprendre. (2e éd.).Bruxelles, Belgique: De Boeck Université.

- Lebrun, M. (2005). E-Learning pour enseigner et apprendre: allier pédagogie et technologie. Louvain-la-Neuve, Belgique: AcademiaBruylant.

- Livian, Y.F. (2015). La méthodologie de recherche en SHS .Réussir son mémoire ou sa thèse. Centre Magellan - Université Jean Moulin - Lyon 3(en ligne: https://halshs.archivesouvertes.fr/halshs-01102083.Submittedon12Jan.

- Man, S.W. (2004). «Strategies for Educational Blogs ».Temasek Polytechnic. Consulté enseptembre2006: http://www. edublog.net/fles/papers /strategies_for_educational_blogs. pdf

- Ministère de l'Education au Liban. (2015). Mémoire de recherche en sciences de l'éducation ,Université Saint-Joseph ,Faculté des Sciences de l'éducation, (en ligne Guide du mémoire -fsedu. https://fsedu.usj.edu.lb/.../guidemethodologique_Option_Recherche

- Novak, J. D. \& Cañas, A. J. (January 22, 2008). "The Theory Underlying Concept Maps and How to Construct and Use Them, Technical Report IHMC CmapTools 2006-01 Rev 01-2008, Florida Institute for Human and Machine Cognition,, available at: http://cmap.ihmc.us/docs/pdf/ TheoryUnderlyingConceptMaps.pdf.

- O’Donnell, M. (2005). "Blogging as pedagogic practice: Artefact and ecology". Paper presented at Blogtalk Downunder, Sydney, 


\section{Number 70, Part 2, February, 2016}

19-22, Consulté en septembre http://incsub.org/blogtalk/images/Odonnell.doc

- Paoli, C. (2005).Mener des projets en travail collaboratif à distance. Module développé dans le cadre du projet CIIMES, Edition: CIIMES. Univ. de Corse.

- Pillou, J-F.(2007).Applications pédagogiques des weblogs dans le campus virtuel FLE - Université de León, département d'etudes humaines, $\quad$ FILOLOGÍA, $\quad \mathrm{n}^{\mathrm{o}} \quad 29 \quad-\mathrm{pp} .341-373$ (http://flenet.rediris.es/tourdetoile/Tome_BlogsCampusEHO7.html)

- Popper, K. (1979). "La logique des sciences sociales" in Adorno Theodor \& Popper Karl.(1979) De Vienne à Francfort, La querelle allemande des sciences sociales, Bruxelles, Éditions Complexe, pp. 75-90.

- Popper, K. (2013).A la recherche d'un monde meilleur, Paris, les Belles Lettres, pp. 271-272

- Quintin, J-J. (Sep.2012).Approches ET démarches de la recherche en sciences de l'éducation. Master 1sciences de l'éducation ET de la formation, Support de cours, MCF - Université Lumière Lyon 2 www.crea-

tice.org/.../download.php?.../Approches\%20et\%20démarc...

- Quivy, R \& Campenhoudt, L.V. (1995). Manuel de recherche en sciences sociale, 2 e édition, Dunod, Paris.

- Radi, S. (Déc. 2001). Les sciences en Egypte. Ministère des Affaires Etrangères\&), la Commission européenne .France. Paris: IRD.

- Sall, H. (2015) Efficacité ET équité de l'enseignement supérieur. Quels étudiants réussissent à l'Université de Dakar. (Cadre théorique ET méthodologie \& contribution empirique). Dakar. UCAD- Université Cheihk Anta Diop Faculté des Lettres et Sciences Humaines, Département de Philosophie(http://www .fastef-portedu.ucad.sn/cuse/cr/cuzon.htm)

- Sall, H. (2015). Manuel de recherche en technologie éducative. Chaire UNESCO Sciences de l'éducation CUSE/FASTEF/UCAD-Dakar ET équipe Labo CUSE, (https://edutechwiki.unige.ch/fr/Manuel_de_recherche_en_technol ogie_\% $3 \%$ A9ducative.

- Sokal, A. (2013). Qu'est-ceque la science? Quelle importance. Conférence prononcée le 23 mai à l'Ecole Normale Supérieure, Paris

- Stanley, G. (2005). Blogging for ELT. British Council. Consulté en septembre 2006: http://www.teachingenglish.org.uk/think/resources/blogging.shtml.

\section{0}


- Tomé, M. (2007). Le Blog. Récupéré le 13 décembre 2007 du site de Wikipédia: http://fr.wikipedia.org/wiki/Blog.

- Tomé, M. (2007). «Applications pédagogiques des weblogs dans le Campus Virtuel FLE ». Université de Léon. Version électronique: Revue Tourdetoile, $\mathrm{n}^{\mathbf{0}}$ 10. Consulté en mai 2008: http://fenet.rediris.es/tourdetoile/Tome_BlogsCampusEHO7.html

- Tomé, M. (2007). «Expériences pédagogiques dans le Campus Virtuel FLE ». In: D'autres espaces pour les cultures. Etudes de Linguistique Appliquée: Revue de didactologie des languescultures ET de lexiculturologie, $n^{o}$ 146, pp.179-188. Paris: Klincksieck.

- Tomé, M. (2009). «Weblogs éducatifs pour l'enseignement d'une Langue Etrangère". Synergies Espagne. $N^{\circ} 2$ - pp. 261-279.

- Ward, J. M. (2004). «Blog assisted language learning (BALL): Push button publishing for the pupils ». TEFL Web Journal, Vol 3, $n^{o}$ 1. Consulté en septembre 2006: http://www.tefwebj.org/v3n1/blog_ward.pdf

\section{潾潾潾潾}

